\title{
氧胺类化合物的合成及其应用研究进展
}

\author{
黎吉辉 $*, a, b$ 李正章 $c$ 张玉苍 $a, b$ 许文茸 $a, b$ 徐树英 ${ }^{a, b}$ \\ ( ${ }^{a}$ 海南大学热带岛屿资源先进材料教育部重点实验室 海口 570228) \\ ( ${ }^{b}$ 海南大学材料与化工学院 海口 570228) \\ ( ${ }^{c}$ 海南出入境检验检疫局技术中心 海口 570100)
}

\begin{abstract}
摘要 氰胺类化合物 $\left(\mathrm{R}^{1} \mathrm{R}^{2} \mathrm{NCN}\right)$ 是一种带有氨基和氰基双官能团的重要精细化工原料，广泛应用于医药、农药、化肥、 保健产品和材料等领域, 受到合成化学家和药物化学家们的广泛关注. 近年来对于氰胺合成方法和其反应的研究发展 迅速，不断有新的氰胺合成方法和以其为原料合成各种含氮化合物的反应涌现. 本文主要综述了近二十年来发展的氰 胺合成方法及氰胺在有机合成中的应用，全面介绍氰胺的各种合成方法和反应，归纳总结他们的特点、规律和优劣势， 为氰胺的合成方法和反应性的研究提供帮助.
\end{abstract}

关键词 氯胺; 合成方法; 反应性; 含氮化合物

\section{Progress on the Synthesis and Applications of Cyanamides}

\author{
Li, Jihui*,a,b \\ Li, Zhengzhang ${ }^{c}$ \\ Zhang, Yucang ${ }^{a, b}$ \\ $\mathrm{Xu}$, Wenrong ${ }^{a, b}$ \\ Xu, Shuying ${ }^{a, b}$ \\ $\left({ }^{a}\right.$ Key Laboratory of Ministry of Education for Advanced Materials in Tropical Island Resources in \\ Hainan University, Haikou 570228) \\ $\left({ }^{b}\right.$ College of Materials and Chemical Engineering, Hainan University, Haikou 570228) \\ ( ${ }^{c}$ Inspection Quarantine Technology Center of Hainan Entry-Exit Inspection Quarantine Bureau, Haikou 570100)
}

\begin{abstract}
Cyanamides are an important class of fine chemicals containing amino and cyano functionalities, which have been widely used for the synthesis of pharmaceuticals, agricultural chemicals, health products and materials, and attracted considerable attention from both organic synthetic chemists and medicinal chemists. Great advances in the synthesis and transformations of cyanamides were made, a diversity of synthetic methods and transformations of cyanamides were developed in the past two decades. In this paper, various synthetic methods and reactions of cyanamides are introduced comprehensively, their characteristics, rules, advantages and disadvantages are also summarized and discussed for the development of new synthetic methods and reactions of cyanamides.

Keywords cyanamides; synthetic methods; reactivities; nitrogen compounds
\end{abstract}

氰胺 $\left(R^{1} R^{2} N C N\right)$ 为一类重要的二氮化合物, 具有多 样性生物活性, 如杀虫活性、杀菌活性 ${ }^{[1]}$ 、抗组织蛋白 酶活性 ${ }^{[2]}$ 和抗磷酸二酷酶. 4(PDE4)活性 ${ }^{[3]}$ 等, 广泛应用 于农药和医药等领域. 另外, 氧胺是一种重要的化工原 料和有机合成药物中间体 ${ }^{[4]}$, 同时也是一种良好的金属 配体 ${ }^{[5]}$.

由于氰胺在农药、医药、染料和有机合成等领域中 都有广阔的应用前景, 引起了有机合成化学家和药物合 成化学家的广泛关注. 近 20 年来, 合成化学家们对氰胺 的合成方法及其在有机合成中的应用展开全面研究, 取
得了丰硕的研究成果.

最简单的氰胺为 $\mathrm{NH}_{2} \mathrm{CN}$, 是一种重要的有机化工 中间体和医药原料, 可通过尿素在 $\mathrm{Ca}(\mathrm{OH})_{2}$ 促进下高温 脱水反应制备得到，该方法已实现工业化 ${ }^{[6]}$. 随着氧胺 在不同领域应用的推广和深入, 对各种氧胺类化合物的 需求也不断提高, 促进了氰胺类化合物合成方法的发 展. 近年来, 各种氰胺类化合物新的合成方法层出不穷, 特别是一些高效、低毒环保的合成方法. 同时, 氧胺合 成方法的发展有力地推动氧胺反应活性的研究, 一系列 反应被发现并广泛应用于合成各种含氮类化合物，拓展

\footnotetext{
* Corresponding author. E-mail: lijihui@hainu.edu.cn

Received June 2, 2017; revised June 26, 2017; published online July 14, 2017.

Project supported by the Natural Science Fundation of Hainan Province (Nos. 20162015, 217008).

海南省自然科学基金(Nos. 20162015, 217008)资助项目.
} 
了氰胺在有机合成中的应用. 本文主要综述了近 20 年 来氰胺类化合物的合成方法及其在有机合成中的应用 研究进展.

\section{1 氰胺的合成}

早期, 合成氰胺的方法主要基于 $\mathrm{NH}$-胺 $\mathbf{1}$ 和氰化溴 (2)的亲核取代反应 ${ }^{[7]}$ (Eq. 1)及 von Braun 反应 ${ }^{[8]}$ (Eq. 2). 后来 Parry 等 $^{[9]}$ 又发展了固载化的胺和氰化溴的反应实 现固相合成氰胺. 这些方法原料简单易得、操作简便, 但存在比较突出的缺陷, 以剧毒的氰化溴为氰基化试 剂, 且反应受电子和位阻效应影响严重, 位阻大或者贫 电子的胺, 反应产率低.

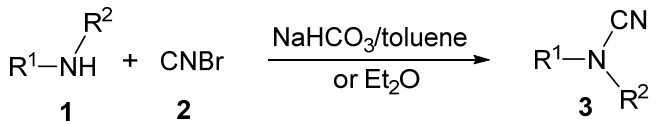

$$
\begin{aligned}
& \mathrm{R}_{4}^{1-N_{\mathrm{R}^{1}}^{\prime}}+\underset{\mathbf{2}}{\mathrm{R}^{1}}+\underset{5}{\mathrm{CNBr}} \stackrel{\Delta}{\mathrm{R}^{1}-N^{\prime}}
\end{aligned}
$$

由于氰化溴的剧毒性，限制了上述合成方法的推广 应用，因此发展以低毒性化合物为原料的合成方法已成 为氧胺合成亟待解决的问题. 为此, 近十年来合成化学 家们发展了各种以低毒性化合物为原料的合成方法.

2009 年 Satyanarayan 等 ${ }^{[10]}$ 以氨基二硫甲酸的铵盐 6 为起始原料, 通过 “一锅者” 三步反应直接合成氰胺 7 (Eq. 3). 氨基二硫甲酸的铵盐首先在三乙胺和碘作用下 发生还原脱硫反应生成异硫氰酸酯, 异硫氧酸酯接着和 氨进行亲核加成和还原脱硫反应生成氰胺. 该反应避免 使用高毒性的氰基化合物为原料，是较环保的合成方 法，但该反应只适用于合成一取代氰胺.

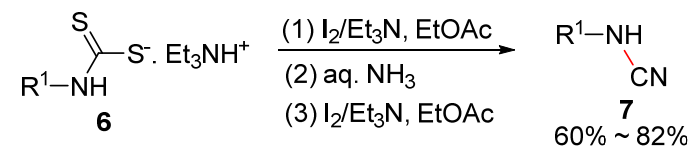

2010 年, Punniyamurthy 等 ${ }^{[11]}$ 报道了 $\mathrm{CuSO}_{4} \cdot 5 \mathrm{H}_{2} \mathrm{O}$ 催 化 2-碘芳基硫嫝(8)和芳基碘(9)串联反应，高效合成 2芳基硫代芳基氰胺(10) (Scheme 1, a). 作者提出以下反 应机理: 首先, 2-卤代芳基硫脲在铜催化下进行分子内 $\mathrm{C}-\mathrm{S}$ 偶联反应生成噻唑 $\mathbf{A}$. 接着, 芳基碘和铜发生氧化 加成反应, 并进一步和噻唑进行反应产生中间体 B. 最 后 B 发生还原消除反应即可生成目标产物. 几个月后, Patel 课题组 ${ }^{[12]}$ 又意外发现 CuI 催化 2-卤代芳基硫嫝(11) 反应合成芳基氰胺类化合物(10) (Scheme 1, b). 在配体 协助下, CuI 催化 2-卤代芳基硫脲和碘代芳烃反应生成 2-芳基硫代芳基氰胺. 该催化体系对底物具有良好的兼 (a)

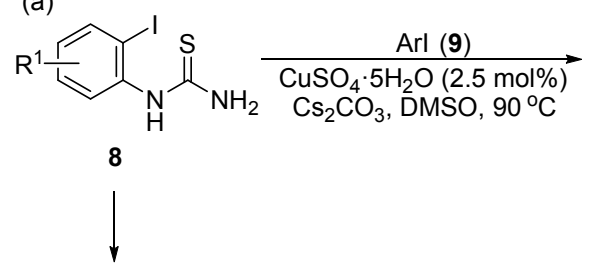<smiles>N#[N+]c1cc[R]#cc1Br</smiles>
$83 \% \sim 93 \%$<smiles></smiles>
$\stackrel{\mathrm{Arl} / \mathrm{CuSO}_{4} \cdot 5 \mathrm{H}_{2} \mathrm{O}}{\longrightarrow}$<smiles>N=C=Nc1cc[R1]([Al])cc1SC[Al][Ba]</smiles>

(b)<smiles>[X]c1c[R]#ccc1NC#N</smiles>

12 $52 \% \sim 98 \%$

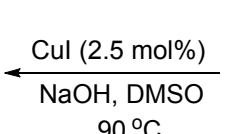

$90^{\circ} \mathrm{C}$

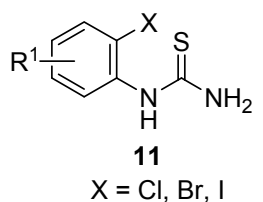

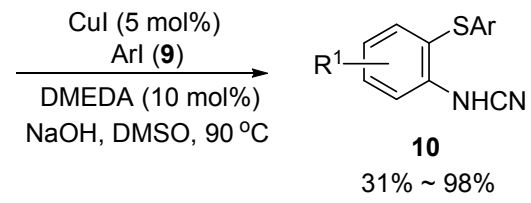

图式 1 铜催化 2-卤代芳基硫艮串联反应合成芳基氰胺 Scheme 1 Copper-catalyzed cascade reaction of 2-haloarylthioureas for the synthesis of arylcyanamides

容性, 2-碘代和 2-溴代芳基硫嫝都能适应反应. 此外，他 们还发现，在无配体条件下 $\mathrm{CuI}$ 催化 2-卤代芳基硫嫝的 反应则直接发生脱硫反应生成 2-卤代芳基氧胺 $\mathbf{1 2}$.

2014 年, Chen 等 ${ }^{[13]}$ 以毒性稍小的三甲基氰硅烷 (TMSCN)为氰基化试剂, 发展了 $\mathrm{NaOCl} / \mathrm{H}_{2} \mathrm{O}$ 促进二级 胺 $\mathbf{1}$ 的亲电氰基化反应制备二取代氧胺 $\mathbf{3}$, 反应能够生 成中等偏上的产率(Scheme 2). TMSCN 先和 $\mathrm{NaOCl}$ 及 $\mathrm{H}_{2} \mathrm{O}$ 发生反应原位生成氰基氯(A)，接着和胺发生亲核 取代反应产生目标产物。

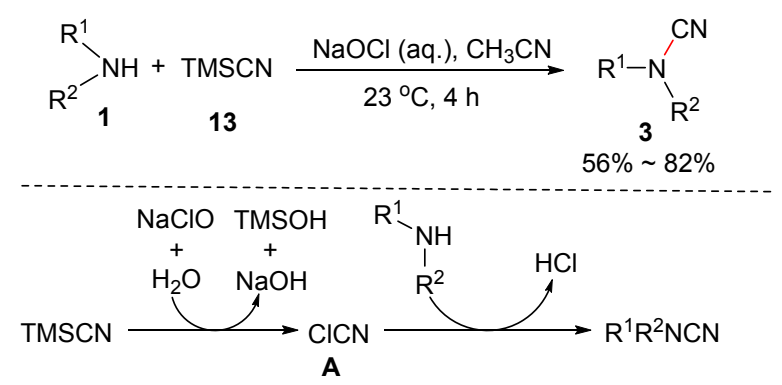

图式 2 胺和 TMSCN 偶联反应合成氰胺 Scheme 2 Coupling reaction of amines and TMSCN for the synthesis of cyanamides

2014 年 Chien 等 ${ }^{[14]}$ 发展了氰 14 和羟胺 15 的两步一 锅煮反应合制备一取代氰胺 16, 反应生成 51\% 95\%的 产率(Eq. 4). 差胺和氰基化合物首先进行亲核加成反应 生成脒肜，接着在碱和磺酰氯的作用下发生 Tiemann 重 
排脱水生成氰胺. 作者还发现咪肟的 Tiemann 重排和水 解一锅煮反应，实现对脲类化合物的合成.

$$
\underset{14}{\mathrm{R}^{1}-\mathrm{CN}} \stackrel{\text { (1) } \mathrm{NH}_{2} \mathrm{OH} \text { (15), EtOH, reflux, } 3 \mathrm{~h}}{\underset{(2) \mathrm{TsCl} \text { or NsCl, DIPEA}, \mathrm{CH}_{2} \mathrm{Cl}_{2}}{\longrightarrow}} \underset{\substack{16 \\ 51 \% \sim 95 \%}}{\mathrm{C}} \stackrel{\mathrm{CN}}{\mathrm{R}}
$$

2016 年 Morrill 等 ${ }^{[15]}$ 以胺 1 和三氯乙氰 17 为原料, 通过一锅煮两步反应实现对氭胺 3 的直接合成(Eq. 5). 胺和三氯乙氰先进行亲核加成, 接着在在碱性条件下发 生脱氯仿反应生成二取代氰胺. 该反应可生成中等偏上 的产率，但底物适用范围窄，只有二级胺适应于该反应. 此外，作者还将反应应用于合成具有抗 PDE4 活性的㲵 胺.

$$
\begin{aligned}
& \underset{1}{\mathrm{R}^{2}-\mathrm{NH}} \stackrel{(1) \mathrm{CNCCl}_{3} 17}{\underset{(2) \mathrm{NaOt}-\mathrm{Am}}{\longrightarrow}} \mathrm{R}^{1}-\mathrm{N}_{3}^{-\mathrm{CN}} \\
& 41 \% \sim 88 \%
\end{aligned}
$$

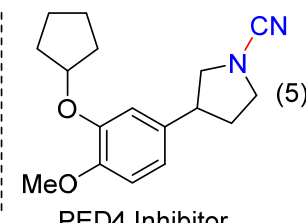

过渡金属催化多组分反应同样也已被应用于合成 氰胺类化合物, 2001 年 Yamamoto 课题组 ${ }^{[16]}$ 发展了钯催 化异氰 18 、三甲基硅叠氮 19 和烯丙醇酯 20 三组分反应 高效合成氰胺 21 (Scheme 3). 反应可以实现一步构建多 个化学键, 高产率生成芳基烯基氰胺. 同时, 反应受底 物的电子和位阻效应影响小, 且对㲵基、酯基和氯等官 能团都有良好的兼容性. 基于实验结果，作者提出了以 下反应机理: 烯丙醇酯、三甲基硅叠氮和钯催化剂先反 应生成叠氮 $\pi$-烯丙基钯 $\mathbf{A}$. 叠氮 $\pi$-烯丙基钯接着和异氧 反应生成 $\pi$-烯丙基钯络合物 B, B 发生类似 Curtius 重排 的反应释放出氮气产生中间体 $\mathbf{C}, \mathbf{C}$ 转化成互变异构体 $\mathbf{D}$ (或 $\mathbf{E}$ ). 最后, $\mathbf{D}$ (或者 $\mathbf{E}$ )发生还原消除反应即可生成目 标产物和钯催化剂.

接下来, 他们以邻位炔基化的异氰苯 $\mathbf{2 2}$ 为原料, 在 高温条件下, 成功将反应应用于合成 $N$-氰基吲哚类化 合物(Scheme 4) ${ }^{[17]}$. 该反应可以实现一步构建多个化学 键直接合成吲哚类化合物. 其反应过程和非邻位炔基的 异氰反应相似，经过多步反应产生中间体 $\mathbf{B} ， \mathbf{B}$ 接着发 生连续的分子内碳碳三键氨基钯化和还原消除反应生 成最终产物。

后来 Yamamoto 课题组 ${ }^{[18]}$ 又发现钯催化异氰 24 和 三甲基硅叠氮(19)的反应也可以直接合成一取代氰胺 25 (Scheme 5). 该反应可应用于合成芳基氰胺和脂肪族氭 胺, 生成中等偏高的产率, 对酯、氰基、炔基等官能团
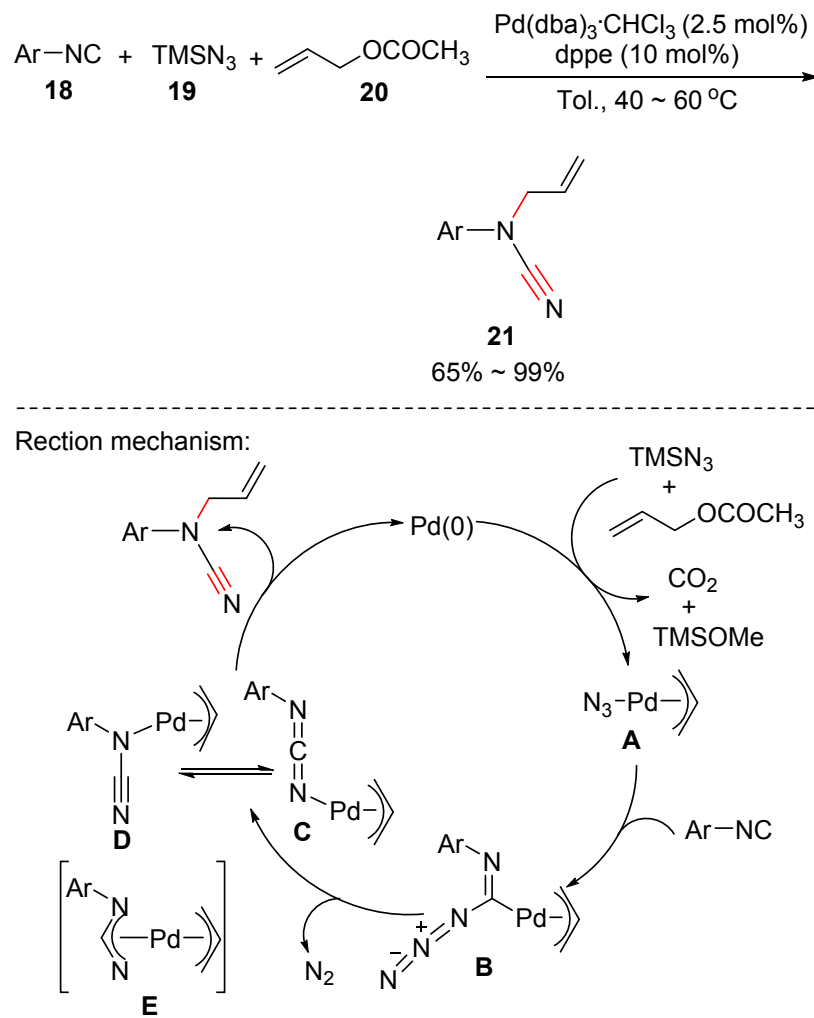

图式 3 钯催化异氰、三甲基硅叠氮三组分反应合成氰胺 Scheme 3 Palladium-catalyzed three-component reaction of isocyanides, allyl carbonate and trimethylsilyl azide for the synthesis of cyanamides
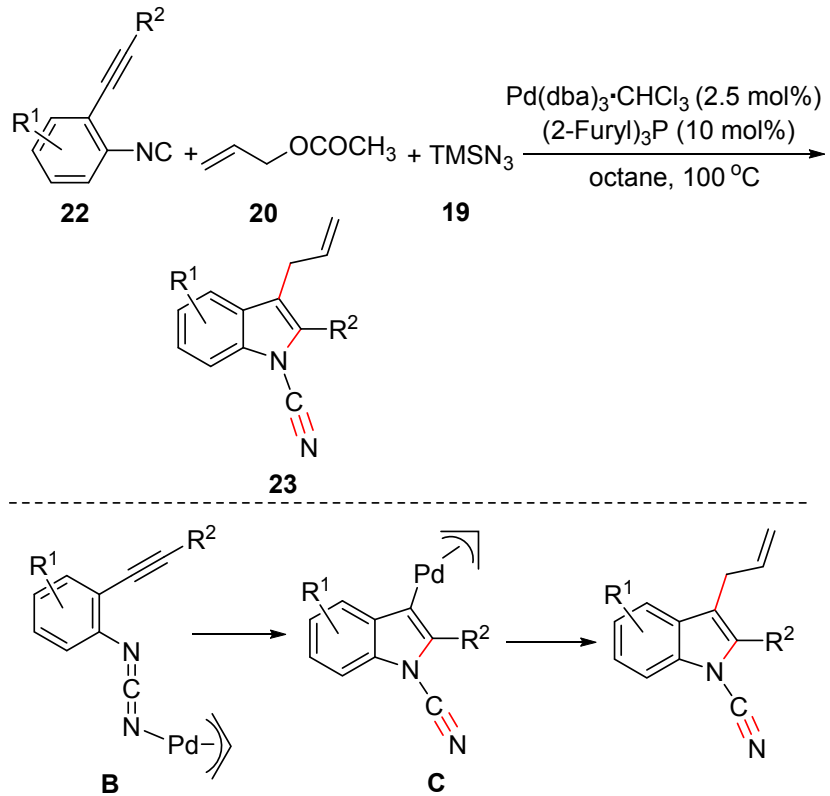

图式 4 钯催化邻炔基芳基异氧、烯丙醇酯和三甲基硅叠氮三 组分反应合成 $N$-氰基吲哚

Scheme 4 Palladium-catalyzed three-component reaction of 2-alkynylaryl isocyanides, allyl carbonate and trimethylsilyl azide for the synthesis of $\mathrm{N}$-cyanoindoles 


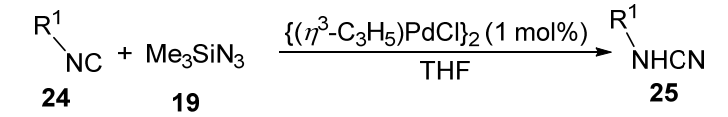

$68 \% \sim 94 \%$

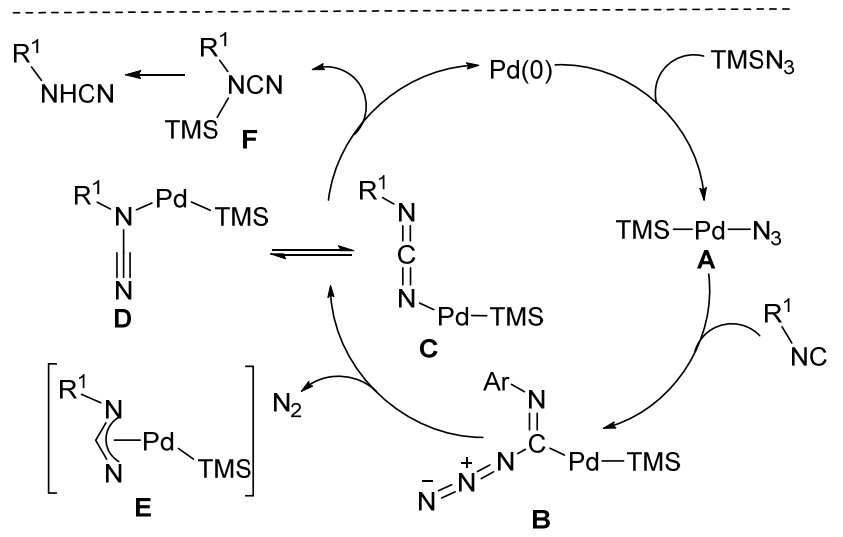

图式 5 钯催化异氰和三甲基硅叠氮反应合成氧胺

Scheme 5 Palladium-catalyzed reaction of isocyanides and trimethylsilyl azide for the synthesis of cyanamides

都具有良好的兼容性. 反应可能经历以下反应历程: 钯 和 $\mathrm{TMSN}_{3}$ 发生氧化加成反应产生钯络合物 $\mathbf{A}$, 再和异 氧进行加成反应生成中间体 B. B 发生类似 Curtius 重排 的反应释放出氮气产生 $\mathbf{C}, \mathbf{C}$ 转化成互变异构体 $\mathbf{D}$ (或 $\mathbf{E}$ ), $\mathbf{D}($ 或 $\mathbf{E})$ 发生还原消除产生 $\mathbf{F}, \mathbf{F}$ 最后在硅胶柱色谱纯化 过程中脱去三甲基硅生成目标产物.

除了上述构键氧胺官能团的合成方法, $N, N$-二取代 氭胺还可通过 $\mathrm{NH}$-氰胺和碘化物的偶联反应快速合成.

2012 年, Louie 等 ${ }^{[19]}$ 证实钯催化 $\mathrm{NH}$-氧胺 $\mathbf{2 6}$ 和卤代 烃类 $\mathbf{2 7}$ 的 $\mathrm{C}-\mathrm{N}$ 偶联反应可以简便合成二芳基氰胺 $\mathbf{2 8}$ (Eq. 6). 芳基氰胺和脂肪族氰胺都适应反应, 生成中等 偏上的产率. 反应受卤代芳烃的位阻效应影响较大, 大 位阻 2-甲基苯基溴和 2,4,6-三甲基苯基澳的反应没有任 何的产物生成.

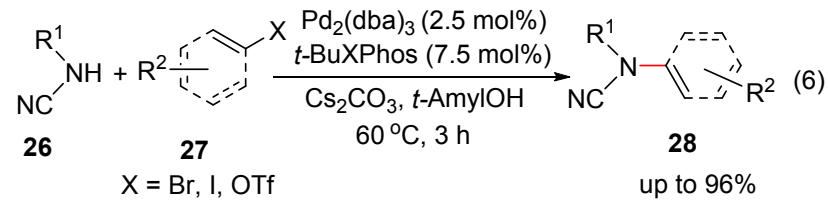

后来, Cui 等 ${ }^{[20]}$ 发现芳香氰胺(29)和六氟磷酸二芳 基碘盐(30)可以在碱性无过渡金属条件下发生 $\mathrm{C}-\mathrm{N}$ 偶 联反应生成二取代氰胺 31 (Scheme 6). 反应在室温下进 行, 条件温和, 但为了避免氰胺被氧化, 反应需要惰性 气体的保护. 另外, 作者还发现氰胺可以先和六氟磷酸 二芳基碘盐进行反应生成芳基化氰胺, 芳基化氰胺接着 在酸和铜共同作用下和三氟甲磺酸二芳基碘盐及 $\mathrm{H}_{2} \mathrm{O}$ 发生三组分反应实现一锅者合成多取代脲类化合物 32.

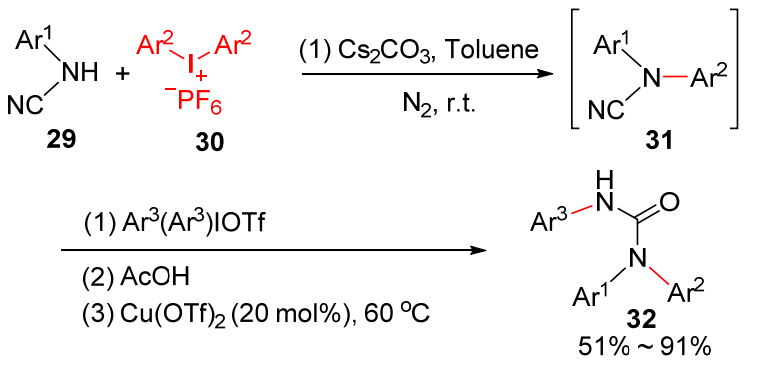

图式 6 氰胺和二芳基碘盐偶联反应合成氰胺和艮 Scheme 6 Cross coupling of NH-cyanamides and diaryliodoniums for the synthesis of cyanamides and ureas

最近, 我们课题组 ${ }^{[21]}$ 证实 $\mathrm{NH}$-氰胺 33 和三氟甲磺 酸二芳基碘盐 34 的 $\mathrm{C}-\mathrm{N}$ 偶联反应可以在水溶液中实 现，高效合成二取代氰胺 35 (Eq. 7). 该反应操作间单， 不需要惰性气体的保护. 此外, 底物适用范围广, 芳基 氭胺和脂肪族氭胺都可以发生反应，芳基氭胺的产率普 遍比脂肪族氰胺的高.

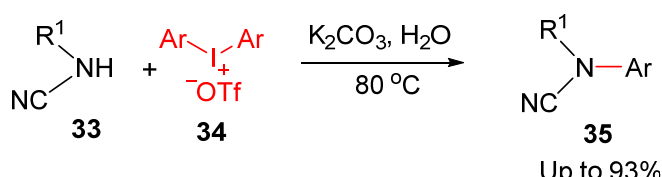

\section{2 氭胺的应用}

氰胺含有氨基和氧基两种活性基团, 兼具亲核和亲 电的反应性能，易发生加成、取代、缩合等反应，是一 类多功能有机合成中间体，可应用于合成多种含氮类化 合物. 下面我们将对氰胺在有机合成中的应用进行详细 介绍.

\section{1 胍及其衍生物的合成:}

以氰胺为原料, 其和亲核试剂的加成反应已被应用 于合成在医药、农药和材料等领域有广泛应用价值的胍 及其衍生物。

早期, 氧胺和亲核试剂的加成反应主要应用于链状 胍、异脲、硫代异腿的合成，这类反应具有 $100 \%$ 的原 子经济性 ${ }^{[22]}$. 后来, 合成化学家们将其推广到环胍及其 衍生物的合成中, 其中 Looper 课题组 ${ }^{[23]}$ 做了系统的研 究工作. 2009 年, 他们结合亲核加成和分子内的氢胺化 反应，发展了炔丙基氰胺 36 和胺 $\mathbf{3 7}$ 的串联反应直接合 成 2-氨基咪唑 38 (Eq. 8). 在 $\mathrm{La}(\mathrm{OTf})_{3}$ 催化下, 胺和炔丙 基氰胺首先发生亲核加成反应生成链状胍, 链状胍接着 进行分子内炔氢氨化反应生成 2-氨基咪唑. 2010 年，他 们成功将反应拓展到 2-硫基咪唑 40 和 2-氧基咪唑 $\mathbf{4 2}$ 的 合成 ${ }^{[24]}$. 在碱促进作用下, 炔丙基氧胺即可以分别和硫 醇 39 及醇 41 发生串联反应生成 2-硫基咪唑(Eq. 8)和 2氧基咪唑(Eq. 10). 
<smiles>[R]Cc1nc(N([R])[R1])n([R18]([H])([H])[18OH])c1[R]</smiles><smiles>[R]Cc1nc(S[R1])n([R1])c1[R]</smiles>

$36 \mathrm{~N}$ 39 40 $53 \% \sim 94 \%$<smiles>[R]Cc1nc(O[R])n([R])c1[R]</smiles>

2015 年 $\mathrm{Fu}$ 等 ${ }^{[25]}$ 同样基于氭胺的加成反应，发展了 铜催化氰胺 43 和邻卤代芳胺 44 的多米诺反应合成稠环 胍 45 (Eq. 11). 氰胺首先和邻卤代芳烃发生 $\mathrm{C}-\mathrm{N}$ 偶联 反应, 接着再进行分子内连续的亲核加成和氢氨化反应 即可实现对稠环胍的构建.

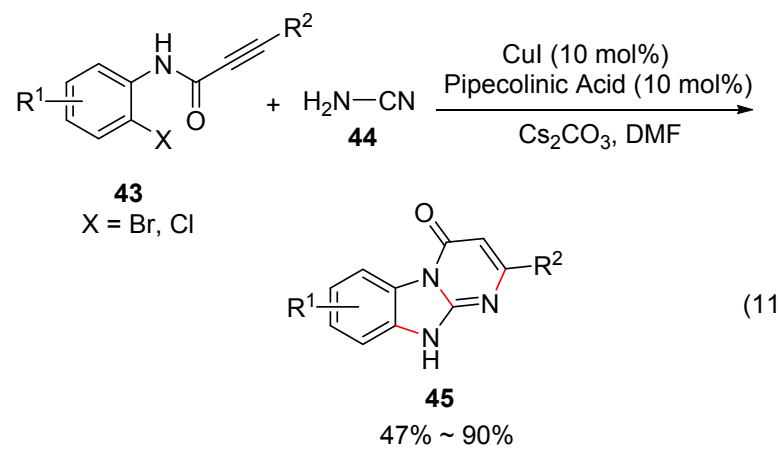

最近, Rassadin 等 ${ }^{[26]}$ 以氰胺 46 为原料, 发展了和吡 啶 $N$-氧 47 的无溶剂多米诺反应, 高效合成脲 48 (Scheme 7). 在甲磺酸的促进作用下, 氰胺和吡啶 $N$-氧 化物先发生亲核加成, 接着进行一系列重排反应生成 $N$-(2-吡啶基)嫝. 该反应的原子利用率为 $100 \%$, 且可在 无溶剂条件下进行, 是一种绿色环保的嫝类化合物的合 成方法.

除了㲵胺的亲核加成反应，近年来研究发现其自由 基加成反应也可以实现，并成功应用到多种胍类化合物 的合成中.

2010 年, Malacria 和 Lacôte 等 ${ }^{[27]}$ 证实 $N$-酰基氰胺 49 在 $\mathrm{Bu}_{3} \mathrm{SnH} / \mathrm{AIBN}$ 促进下引发自由基串联反应高效合 成稠环胍 50 (Scheme 8). 在 $\mathrm{Bu}_{3} \mathrm{SnH} / \mathrm{AIBN}$ 促进下,

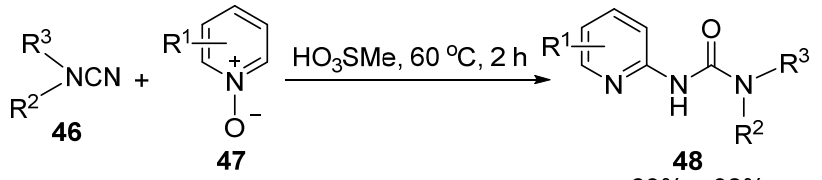
$63 \% \sim 92 \%$

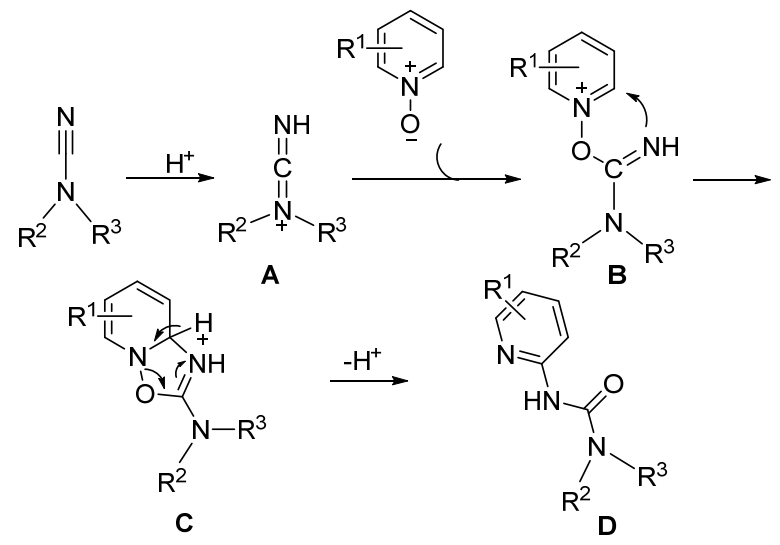

图式 7 氰胺和吡啶 $N$-氧多米诺反应合成艮类化合物

Scheme 7 Domino reaction of cyanamides and pyridine $\mathrm{N}$-oxides for the synthesis of ureas

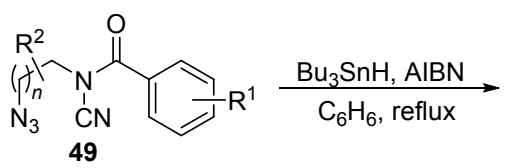<smiles>[R]C1C2=Nc3ccccc3C(=O)N1C(C#[R])N2</smiles>

50 $24 \% \sim 82 \%$

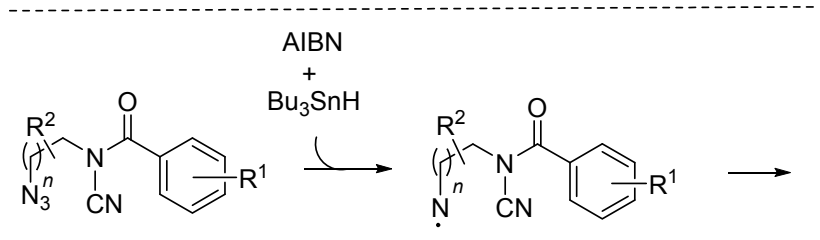

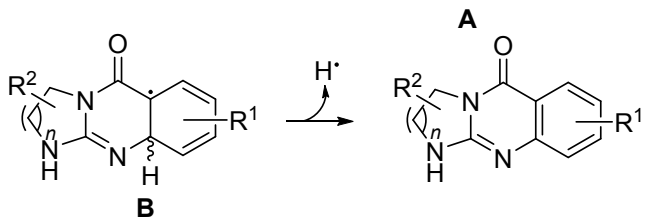

图式 $8 N$-酰基氰胺自由基串联反应合成稠环胍

Scheme 8 Radical cascade reaction of $N$-acyl cyanamides for the synthesis of fused cyclic guanidines

叠氮基先生成氨基自由基，接着发生串联的自由基加成 和 $\mathrm{C}-\mathrm{N}$ 偶联反应产生稠环胍.

两年后, Malacria 和 Lacôte 等 ${ }^{[28]}$ 又发现 $N$-酰基氰胺 51 和芳香二硫 52 在 Hünig 碱和光照条件作用下可发生 自由基串联反应合成硫代异脲 $\mathbf{5 3}$ (Scheme 9). 在光照条 件下，芳香二硫化合物和 Hünig 碱发生自由基反应生成 硫醇自由基，接着和氰基发生加成和后续的重排反应生 成硫代异嫝类化合物。此外，基于该反应，他们发 
展了 $N$-酰基氰胺、二硫化合物和胺的一锅煮三组分反 应，实现对胍类化合物的直接合成.

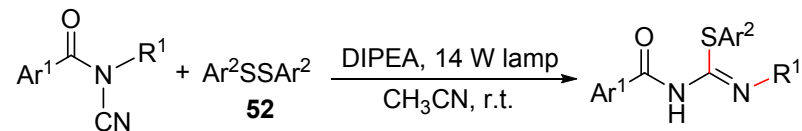

$$
\begin{aligned}
& 51 \\
& 53
\end{aligned}
$$

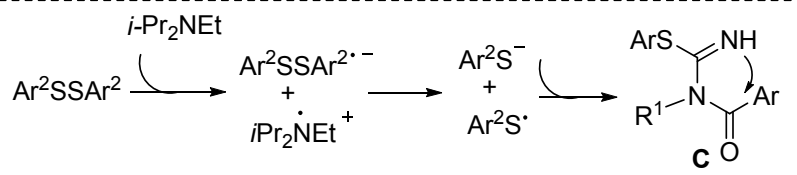

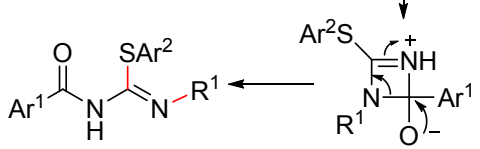

图式 $9 N$-酰基氰胺和二硫化合物自由基串联反应合成异艮 类化合物

Scheme 9 Radical cascade reaction of $N$-acyl cyanamides and diaryldisulfides for the synthesis of isothioureas.

最近 Neuville 和 $\mathrm{Li}$ 等发展了铜催化氰胺 54、脂肪 胺 55 和嗍酸 56 三组分反应高效合成 $N, N^{\prime}, N^{\prime \prime}$-三取代胍 57 (Scheme 10) ${ }^{[29]}$. 由于反应在氧气氛围下进行, 底物 适用范围有一定的局限性且对官能团兼容性差. 作者对 反应机理进行了深入研究, 提出了以下反应机理: 硼酸 先和铜发生金属转移反应生成中间体 A. A 接着和氧胺 进行配位，并在碱作用下发生去质子化和重排生成 $\mathbf{B}^{\prime}$. B'进行氧化、亲核加成和还原消除的串联反应生成
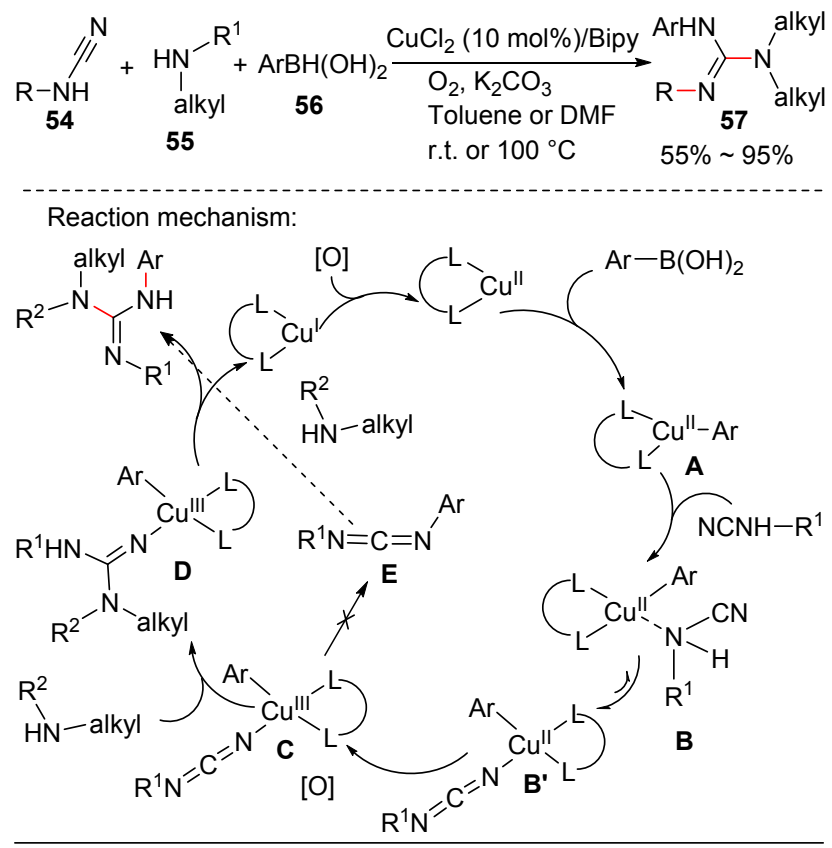

图式 10 铜催化氰胺、胺和嗍酸三组分反应合成胍 Scheme 10 Copper-catalyzed three-component reaction of cyanamides, amines and boronic acides for the synthesis of guanidines
目标产物. 后来，基于该三组分反应，他们通过利用不 同的氰胺为原料, 实现一锅者反应直接合成 2-氨基苯并 咪唑和 2-氨基喹啉 ${ }^{[30]}$.

由于上述铜催化氧胺、胺和硼酸三组分反应的氧化 反应条件对底物和官能团的兼容性较差，且一些硼酸原 料的热稳定差，使得该反应的应用有一定的局限性. 为 了克服上述反应中存在缺点, 最近我们课题组以三氟甲 磺酸二芳基碘盐 58 为芳基化试剂, 发展了铜催化其和 氧胺 $\mathbf{5 4}$ 及胺 $\mathbf{5 5}$ 三组分反应高效构建胍 $\mathbf{5 9}$ (Eq. 12) ( $^{[31]}$. 相交于之前发展的铜催化嗍酸的三组分反应 ${ }^{[30]}$, 本反 应具有更广泛的适用性. 脂肪族和芳香族胺都能适应反 应，但芳香族胺的产率较低. 杂环芳基碘盐同样也适用 于本反应，可用于合成芳杂环取代的胍类化合物. 另外， 具有环张力的环丙基氰胺同样以较好的产率生成相应 环丙基胍. 然而，该反应中二芳基碘盐仅有一个芳基参 与反应，反应原子经济性低.

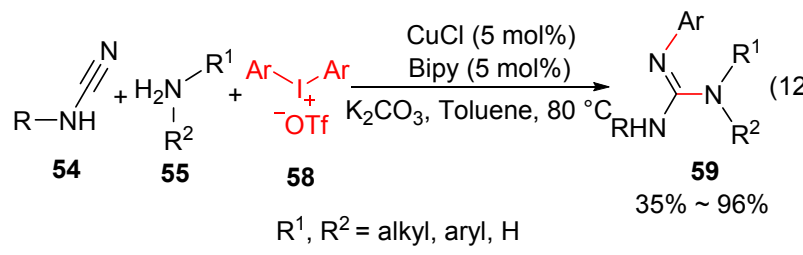

\subsection{2 -氨基氮杂环的合成}

过渡金属催化氰胺和炔的环加成反应是一类高效 的有机反应，具有很高的原子经济性，倍受合成化学家 的青崃，广泛应用于各类环状含氮化合物的合成，如广 泛存在于药物分子中的 2-氨基吡啶和 2-氨基喹啉类化 合物.

过渡金属催化氧胺和炔的环加成反应早在 1978 年 就已经有报道 ${ }^{[32]}$. 随着对其研究的深入, 近年来陆续有 过渡金属催化氧胺和二炔的环加成反应的报道. 2004 年 Maryanoff 等 ${ }^{[33]}$ 报道了钴促进二取代氰胺 $\mathbf{6 1}$ 和二炔 $\mathbf{6 0}$ 的 $[2+2+2]$ 环加成反应，高效合成稠环氨基吡啶 62 (Scheme 11). 以 $\mathrm{CoCp}(\mathrm{CO})_{2}$ 为促进剂，反应在回流的 1,4-二氧六环溶剂中进行，具有 $100 \%$ 的原子经性. 反应 可能经历以下反应历程: $\mathrm{CoCp}(\mathrm{CO})_{2}$ 首先和二炔配位并 释放出 $\mathrm{CO}_{2}$ 生成络合物 $\mathbf{A}, \mathbf{A}$ 接着发生炔氧化加成生成 环钴二烯络合物 B. B 和氧胺发生类似 Diels-Alder 的环 加成反应产生 $\mathbf{C}$, 最后 $\mathbf{C}$ 发生还原消除生成目标产物和 钴催化剂.

Louie 等 ${ }^{[34]}$ 证实以 $\left[\mathrm{Ni}(\mathrm{cod})_{2}\right] / \mathrm{IMes}$ 为催化剂, 可以 实现二取代氧胺 64 和二炔 63 的 $[2+2+2]$ 环加成催化反 应得到 65 (Eq. 13 ). 反应可实现在低催化剂量和室温条 件下进行, 条件比较温和, 对官能团具有良好的兼容性. 反应具有良好的选择性, 不对称二炔反应生成的产物是 氨基在较大基团邻位的稠环吡啶. 此外，作者还证实该 

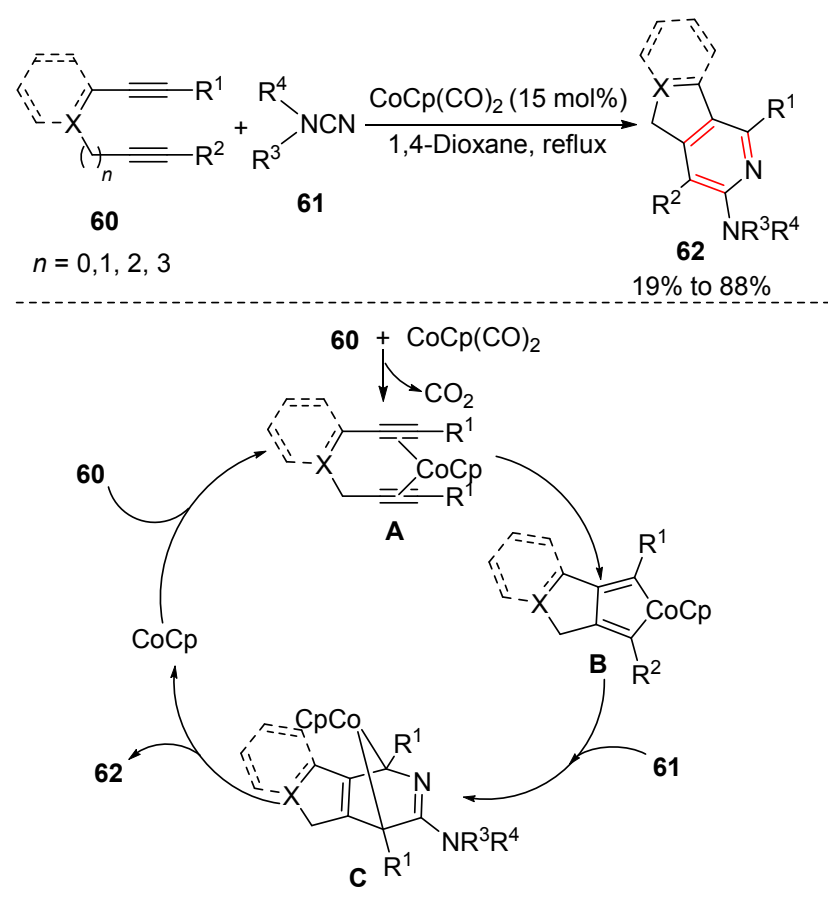

图式 11 钴促进二炔和氰胺 $[2+2+2]$ 环加成反应合成稠环 2氨基吡啶

Scheme 11 Cobalt-promoted $[2+2+2]$ cycloaddition of bis-alkynes and cyanamides for the synthesis of fused 2-aminopyridines

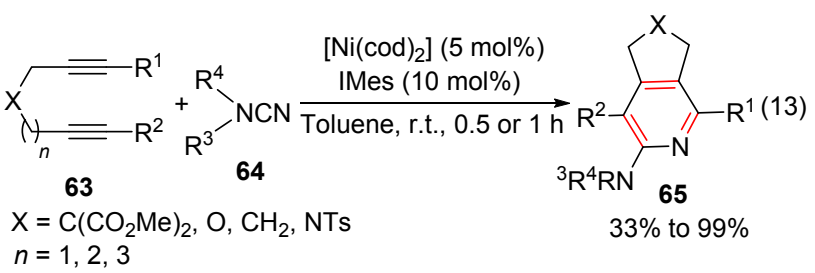

催化体系还适用于氰胺和两分子单炔的反应 ${ }^{[34 a]}$.

后来 Wan 课题组 ${ }^{[35]}$ 和 Louie 课题组 ${ }^{[36]}$ 又分别发现以 廉价的铁为催化剂, 同样可以催化二取代氰胺 67 和二 炔 66 的 $[2+2+2]$ 环加成反应合成氨基稠环吡啶 68 (Eq. 14). Louie 等以 $\mathrm{FeCl}_{2} / \mathbf{L 1}$ 为催化体系, 反应需要在加热 条件下进行; 在该催化体系下, 不对称二炔的反应生成

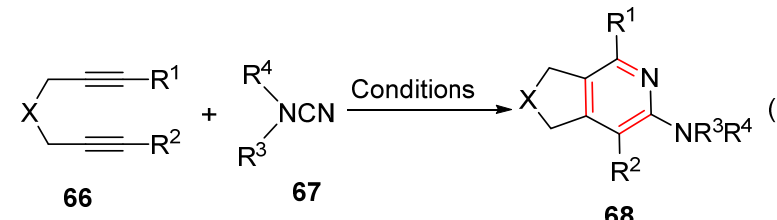

$\mathrm{X}=\mathrm{C}\left(\mathrm{CO}_{2} \mathrm{Me}\right)_{2}, \mathrm{O}, \mathrm{CH}_{2}, \mathrm{NTs}$

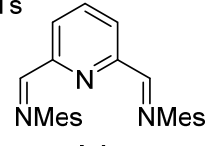

L1

Conditions: (a) $\mathrm{FeCl}_{2}$ (5 mol\%), L1 (10 mol\%), $\mathrm{Zn}$ (10 mol\%), $\mathrm{C}_{6} \mathrm{H}_{6}$, $70^{\circ} \mathrm{C}$ (Janis Louie); (b) $\mathrm{Fel}_{2}$ (5 mol\%), dppp (10 mol\%), Zn (10 mol\%), THF, r.t. (Boshun Wan)
的产物主要是氨基在较小基团邻位的稠环吡啶. Wan 和 合作者则以 $\mathrm{FeI}_{2} / \mathrm{dppp}$ 为催化体系, 可实现反应在室温 下进行; 该催化体系的区域选择性和 $\mathrm{FeCl}_{2} / \mathrm{L} 1$ 的刚好互 补, 不对称二炔反应的产物主要是氨基在较大基团邻位 的稠环吡啶.

一年后, Louie 课题组 ${ }^{[37]}$ 还将铁催化体系应用到氰 胺 70 和炔氰 69 的 $[2+2+2]$ 环加成反应中, 实现对稠环 2-氨基喹啉 71 的合成(Eq. 15). 作者通过反应条件篮选 优化，找出最佳的催化体系为 $\mathrm{FeI}_{2} /{ }^{i-\mathrm{Pr}} \mathrm{PDAI}$, 反应可以 在较低的温度下进行.

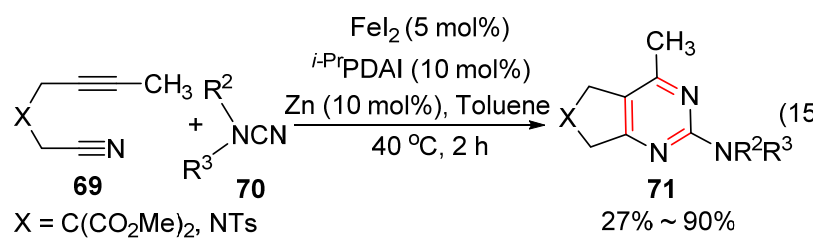

2015 年, Takeuchi 等 ${ }^{[38]}$ 以铱为催化剂, 同样也可以 高效催化二取代氰胺 73 和二炔 72 的 $[2+2+2]$ 环加成反 应，反应适用范围广且对官能团的兼容性强，可以很好 地兼容酯基、羟基和羰基等官能团(Eq. 16). 通过实验证 实，不对称二炔反应的区域选择性主要受电子效应控 制，二炔中富电子的一端容易和㲵基中的碳相结合，而 缺电子的一端则和氰基中的氮相结合. 此外, 作者还发 现氰胺反应的产率普遍比氰反应 ${ }^{[39]}$ 的高, 并通过理论 计算对该现象进行解释.

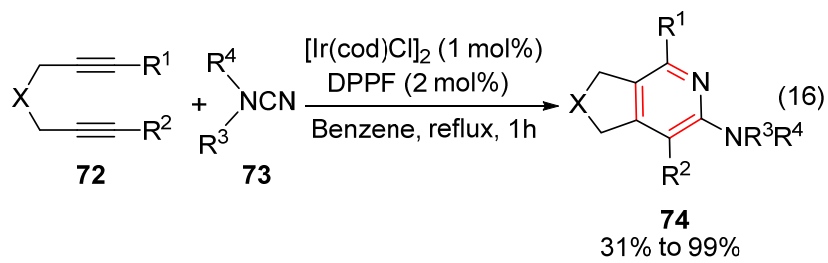

最近, Ratovelomanana-Vidal 和 Michelet 等 ${ }^{[40]}$ 报道了 $\mathrm{Cp} * \mathrm{Ru}\left(\mathrm{CH}_{3} \mathrm{CN}\right)_{3} \mathrm{PF}_{6}$ 催化 $\alpha, \omega$-二炔 75 和二取代氰胺 76 的 $[2+2+2]$ 环加成反应高效合成稠环 2-氨基吡啶 77 (Eq. 17). 反应的条件温和, 对官能团具有良好的兼容 性，能兼容羟基、酯基、氰基和卤素等官能团. 不对称 二炔的反应生成很高的区域选择性，主产物为位阻较小 2-氨基吡啶类化合物.

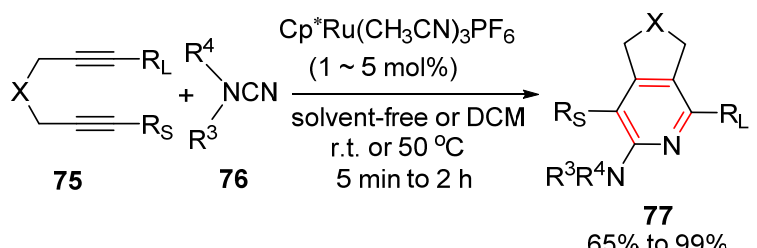


除了和二炔的反应, 2015 年 Rassadin 等 ${ }^{[41]}$ 发现单炔 78 也可以和二取代氰胺 79 及 2 -甲基吡啶氧发生 $[2+$ $2+1$ ]环化反应生成 2-氨基恶唑类化合物 80 (Eq. 18). 反 应以 $\mathrm{Ph}_{3} \mathrm{PAuNTf}_{2}$ 为催化剂和甲磺酸为促进剂, 在中等 温度下进行, 生成中等偏上的产率. 同时, 作者还发现 了氰胺和 2-甲基吡啶氧在甲基磺酸促进下反应生成嫝 类化合物. 作者提出在金催化下炔先和 2-甲基吡啶氧化 物生成 $a$-氧金卡宾中间体 IM-1, 接着和氧胺发生多步 反应产生目标产物.

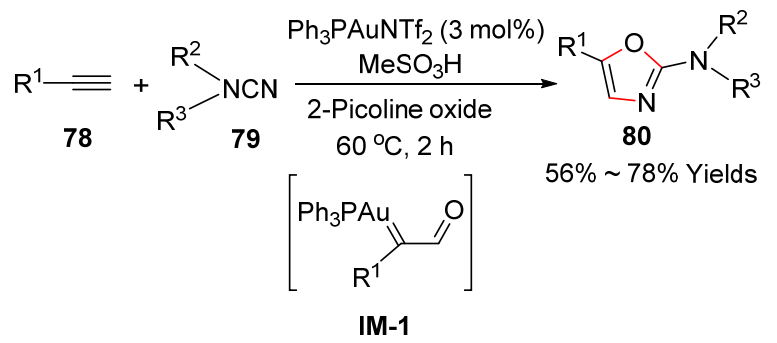

\section{3 氭基化合物的合成}

氧胺类化合物作为一类重要的亲电氧基化试剂, 已 被广泛应用于合成具有高应用价值的氰基化合物. 常见 的亲电氧基化试剂有 1 -氧基苯并三唑 ${ }^{[42]} 、 1$-氰基咪 唑 ${ }^{[43]} 、 N$-氧基丁二酰亚胺、1-氧基苯并咪唑 ${ }^{[44]} 、 N$-氧 基- $N$-苯基对甲苯磺酰胺 $(\mathrm{NCTS})^{[45]}$ 和二烷基氰胺 ${ }^{[46]}$ 等, 其中 $N$-氧基- $N$-苯基对甲苯磺酰胺(NCTS)具有毒性低、 稳定和实用等优点, 近年来对其反应活性的研究异常活 跃.

2011 年, Beller 和 Anbarasan 等 ${ }^{[45 a]}$ 以 NCTS (81) 为氭 基化试剂, 发展了 $\left[\left\{\mathrm{Rh}(\mathrm{OH})(\mathrm{cod})_{2}\right\}\right]$ 催化硼酸 82 的氰基 化反应合成氰类化合物 83 (Scheme 12). 相较于金属氰 化物的亲核氰基化反应，该反应避免了使用剧毒试剂; 但反应中往往产生 $N$-苯胺对苯磺酰胺副产物, 反应的 原子经济性低下. 结合已报道的铑催化芳基硼酸和不饱 和键的加成反应机理，作者推断以下反应过程: 芳基硼 酸和铑发生金属转移生成芳基铑 A, A 接着和 NCTS 配 位产生络合物 B. 然后, B 进行分子内的芳基转移产生 芳基脒络合物 C. 最后, $\mathbf{C}$ 发生还原消除生成芳基氰和 氨基铑化物 $\mathbf{D}, \mathbf{D}$ 在碱性条件和芳基硼酸反应再生成铑 活性催化剂形成催化循环. 同年, 他们又将 NCTS 应用 至和格氏试剂的亲电㲵基化反应中合成各种芳基氰, 反 应中格氏试剂通过镁试剂和芳基溴化物的反应原位生 成 ${ }^{[45 b]} .4$ 年后, Gosmini 等 ${ }^{[45 c]}$ 报道了 $\mathrm{CoBr}_{2} / \mathrm{Zn}$ 促进芳基 溴和 NCTS 的氰基化反应. 作者通过实验证实, $\mathrm{CoBr}_{2}$ 首 先催化芳基溴先和 $\mathrm{Zn}$ 反应生成芳基锌试剂, 接着再催 化芳基锌试剂和 NCTS 进行交叉偶联生成芳基氰. 反应 对酯基、烷硫基、㲵基和酮羰基等活性官能团都有良好 的兼容性.

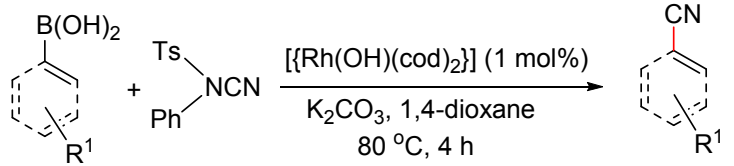

82

81

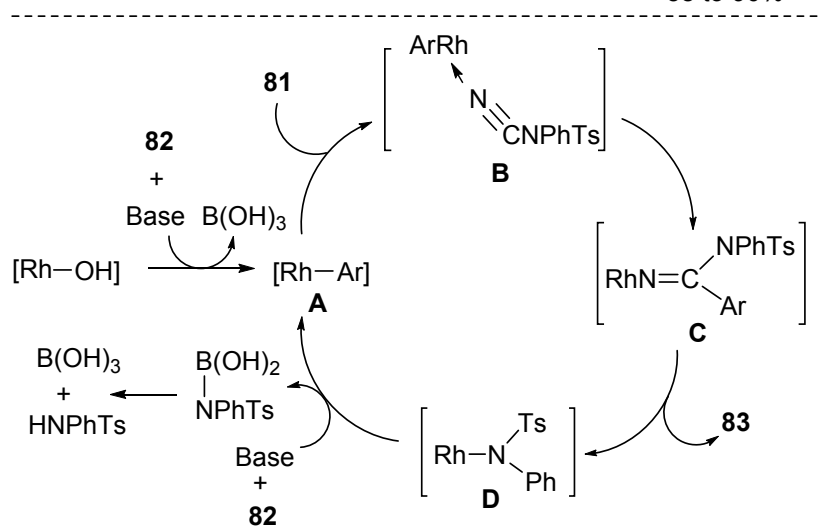

图式 12 铑催化嗍酸和 NCTS 氰基化反应合成氰

Scheme 12 Rhodium catalyzed cyanation of boronic acids with NCTS for the synthesis of nitriles

同样是在 2011 年, Wang 等 ${ }^{[4 \mathrm{~d}]}$ 以 NCTS 为氰基化试 剂，在 $\mathrm{BF}_{3} \cdot \mathrm{Et}_{2}$ 催化下分别和吲哚及吡咯 84 发生 $\mathrm{C}-\mathrm{H}$ 活化/氰基化反应直接合成氰基化合物 85 (Eq. 19). 吲哚 化合物的反应选择性发生 3-位上，而吡咯的反应则主要 发生在 2-位上. 相较于官能化芳烃的反应, 本方法直接 使用简单易得的芳杂烃类化合物为原料，减少对芳烃的 官能化操作，且具有较高的原子经济性.

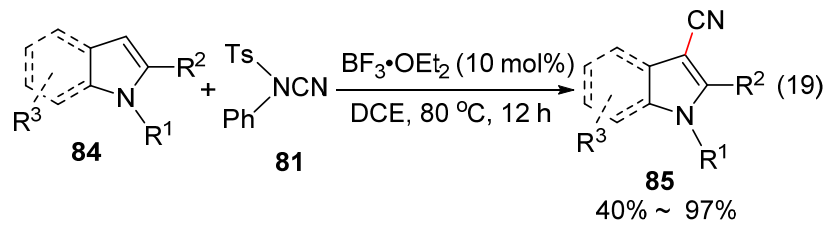

2013 年, Fu 课题组 ${ }^{[45 e]}$ 将 NCTS 应用到芳烃(86) 的导 向氰基化反应中, 成功实现 $\mathrm{C}-\mathrm{H}$ 活化直接合成芳基氧 类化合物 87 (Eq. 20). 作者发现以肟醚、咪唑、吡啶和 吡唑等作为导向基团, 在 $\left[\mathrm{RhCp}^{*}\left(\mathrm{CH}_{3} \mathrm{CN}\right)_{3}\right]\left(\mathrm{SbF}_{6}\right)_{2}$ 的催 化下都可以实现对导向基团邻位 $\mathrm{C}\left(\mathrm{sp}^{2}\right)-\mathrm{H}$ 的活化反应 合成直接邻位氧基化产物. 该反应对官能团具有良好的

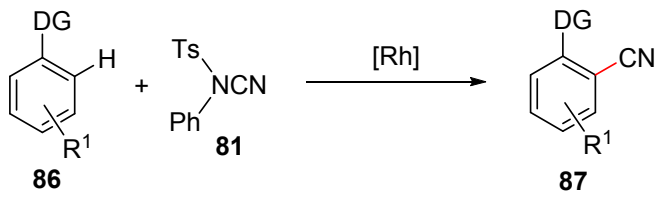

Conditions:

$\left[\mathrm{RhCp}^{*}\left(\mathrm{CH}_{3} \mathrm{CN}\right)_{3}\right]\left(\mathrm{SbF}_{6}\right)_{2}(5 \mathrm{~mol} \%), \mathrm{Ag}_{2} \mathrm{CO}_{3}$, 1,4-dioxane, $120^{\circ} \mathrm{C}, 24 \mathrm{~h}, 53 \%$ to $94 \%$ yields ( $\mathrm{Yao} \mathrm{Fu}$ )

$\left[\mathrm{Cp}^{*} \mathrm{RhCl}_{2}\right]_{2}(1 \mathrm{~mol} \%) / \mathrm{AgSbF}_{6}(10 \mathrm{~mol} \%)$, toluene, $36 \mathrm{~h}$, $23 \% \sim 84 \%$ yields (Pazhamalai Anbarasan) 
兼容性, 羟基、碘、酯、烷硫基和环氧乙环等基团都能 在反应中存活. 几乎在同一时间, Anbarasan 和合作者同 样以 NCTS 为氰基化试剂, 发展了 $\left[\mathrm{Cp}^{*} \mathrm{RhCl}_{2}\right]_{2}$ 催化吡啶 基导向的邻位 $\mathrm{C}\left(\mathrm{sp}^{2}\right)-\mathrm{H}$ 活化氰基化反应合成 2-(2-吡啶 基)苯甲氭类化合物 ${ }^{[45 \mathrm{f}]}$. 这两种 $\mathrm{C}\left(\mathrm{sp}^{2}\right)-\mathrm{H}$ 活化氰基化 反应的发现引起了合成化学家们对过渡金属催化芳烃 和 NCTS 的直接氰基化反应研究的热潮. 近四年来, 以 NCTS 为氰基化试剂, 铑催化不同基团导向的邻位 $\mathrm{C}\left(\mathrm{sp}^{2}\right)-\mathrm{H}$ 活化反应陆续被发现, 如芳基磷酸酯邻位氧 基化反应 ${ }^{[45 \mathrm{~g}]} 、 N$-酰基吲哚啉的 7-氰基化反应、 $N$-(2-嘧 啶基)吲哚的 2-氯基化反应 ${ }^{[45 \mathrm{~h}]} 、 N$-(2-吡啶基)吲哚的 2氯基化反应、 $N$-(2-吡啶基)吡咯的 2-氰基化反应 ${ }^{[45 i]}$ 和芳 基咪唑并 $\left[1,2-\alpha\right.$ 吡啶的双氰基化反应 ${ }^{[45]}$ 等.

除了作为氰基化试剂, 氭胺还被广泛用作氨基氰基 化试剂, 和不饱键发生加成反应合成氰基取代的胺类化 合物.

2016 年 Chien 等 ${ }^{[47]}$ 以铜为催化剂, 催化邻炔基芳香 氰胺 88 的分子内氨基氰基化反应高效合成 3-氰基吲哚 89 (Scheme 13). 作者提出了以下反应机理: 铜先和末 端炔反应生成炔基铜 $\mathbf{A}$, 接着和氰胺基发生 $\mathrm{CN}$ 基转移 反应生成氰基亚烯基铜中间体 $\mathbf{B}$, 最后氨基和氰基亚烯 基铜进行亲核加成和质子化反应生成目标产物.

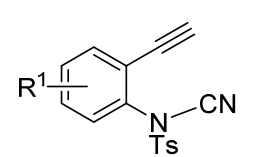

88

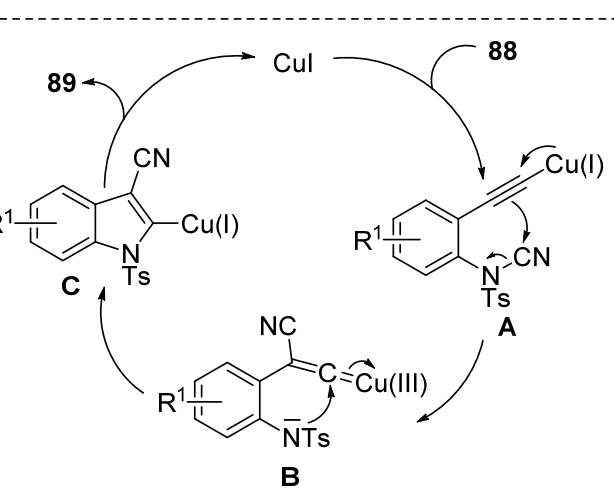

图式 13 铜催化邻炔基芳香氧胺环化反应合成 3-氰基吲哚 Scheme 13 Copper-catalyzed cyclization of $o$-alkynyl arylcyanamides for the synthesis of 3-cyanoindoles

2014 年 Zeng 课题组 ${ }^{[48]}$ 报道了氰胺和苯炔前体的分 子间氨基氰基化反应，高效合成双官能分子 2-氨基苯甲 氰类化合物. 在 $\mathrm{CsF}$ 的促进下, 2-(三甲基硅基)苯酚三氟 甲磺酸酯 $(90)$ 和芳基氰胺 $(91)$ 反应生成 2-氨基苯甲氭类 化合物 92 (Scheme 14). 作者对反应过程进行探索, 并 提出了以下反应机理：2-(三甲基硅基)苯酚三氟甲磺酸
酯在 $\mathrm{CsF}$ 的作用下脱掉三甲基硅基和三氟甲磺酸基生 成苯炔, 苯炔接着和氧胺进行加成及后续的重排和质子 化反应产生 2-氨基苯甲氰.

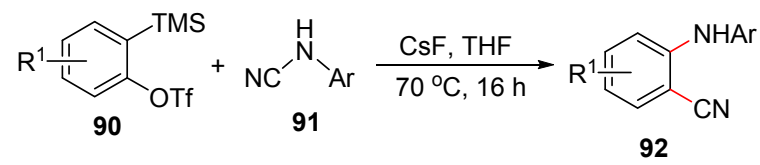
$42 \% \sim 89 \%$

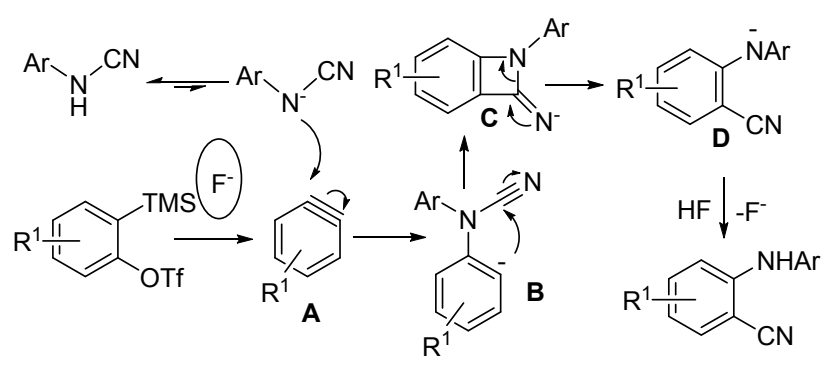

图式 $14 \mathrm{CsF}$ 促进 2-(三甲基硅基)苯酚三氟甲磺酸酯和氰胺 串联反应合成 2-氨基苯甲氰类化合物

Scheme 14 CsF-promoted cascade reaction of cyanamides and (trimethylsilyl)aryl trifluoromethanesulfonate for the synthesis of 2-aminobenzonitriles

除了和炔的氨基氰基化反应，氰胺还可以和烯烃发 生氨基氰基化反应，这类反应已被广泛应用于 $\beta$-㲵基胺 类化合物的合成，具有 $100 \%$ 的原子经济性.

2014 年 Douglas 等 ${ }^{[49]}$ 报道了无金属 Lewis 酸促进 的分子内双键氨基氰基化反应直接合成 2-氰甲基吲哚 啉(94) (Scheme 15). 作者以[2-( $N$-对甲苯磺酰基)氰胺基 苯基]烯烃 $(93)$ 为原料, 在 $\mathrm{B}\left(\mathrm{C}_{6} \mathrm{~F}_{5}\right)_{3}$ 促进作用下, 实现对 氰胺基 C-CN 的切断并和双键发生加成反应构建 2-氰甲
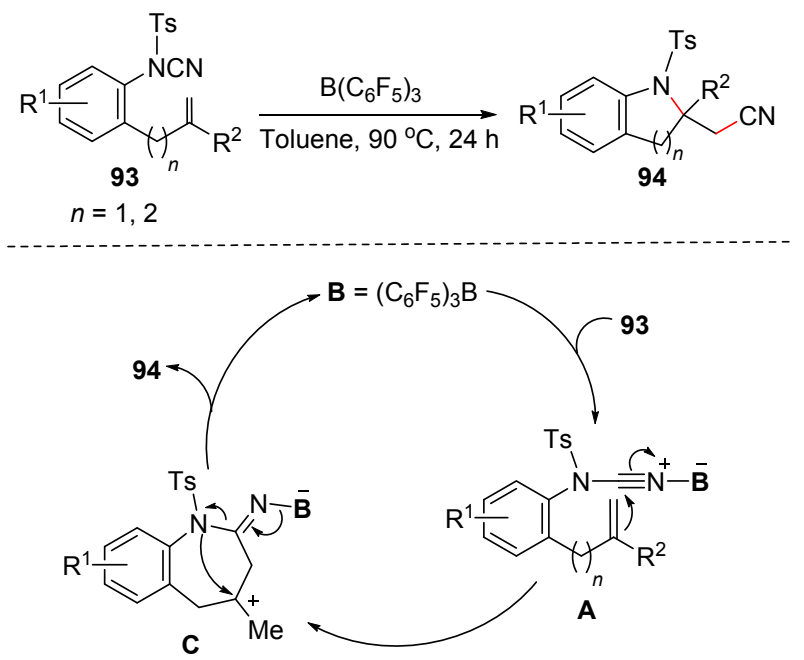

图式 $15 \mathrm{~B}\left(\mathrm{C}_{6} \mathrm{~F}_{5}\right)_{3}$ 促进分子内烯烃氨基氰基化反应合成吲哚 啉

Scheme $15 \quad \mathrm{~B}\left(\mathrm{C}_{6} \mathrm{~F}_{5}\right)_{3}$-promoted intramolecular aminocyanation of alkenes by cyanamides for the synthesis of indolines 
基吲哚啉. 基于实验结果, 作者提出了以下反应机理: 氭胺和 $\mathrm{B}\left(\mathrm{C}_{6} \mathrm{~F}_{5}\right)_{3}$ 配位生成中间体 $\mathbf{A}, \boldsymbol{A}$ 发生烯烃对氭基 碳的亲核进攻产生中间体 C. C 最后发生重排即可生成 最终产物和促进剂 $\mathrm{B}\left(\mathrm{C}_{6} \mathrm{~F}_{5}\right)_{3}$.

后来, Nakao 和其合作者 ${ }^{[50]}$ 又发展了钯催化分子内 烯烃氨基氰基化反应. 他们以 $N$-酰基氰胺(95)为原料, 在 $\mathrm{CpPd}\left(\right.$ allyl)和 $\mathrm{BR}_{3}^{4}$ 的共同作用下发生烯烃氨基氰基 化反应合成吲哚啉 96 (Scheme 16). 基于实验结果, 作 者对反应机理进行了推断: $N$-酰基氰胺和硼烷进行配位 生成 $\mathbf{A}, \mathbf{A}$ 的氰氨基接着和 $\left[\mathrm{Pd}^{0}\right]$ 发生氧化加成生成 $\mathbf{C .} \mathbf{C}$ 进行 exo-trig 顺式氨基钯化反应产生 D. D 最后发生还原 消除反应和嗍烷转移反应生成目标产物、 $\left[\mathrm{Pd}^{0}\right]$ 催化剂和 络合物 $\mathbf{A}$ 形成催化循环.
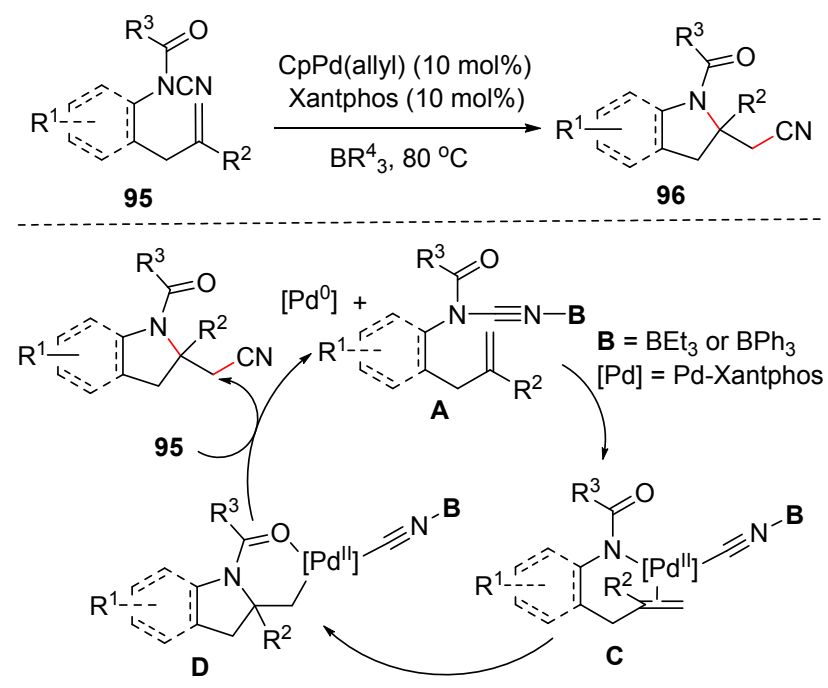

图式 16 钯催化分子内氨基氰基化反应合成吲哚啉 Scheme 16 Palladium-catalyzed intramolecular aminocyanation of alkenes for the synthesis of indolines

2013 年 Wang 等 ${ }^{[51]}$ 发展了铑催化芳基乙烯 97 的分 子内 $\beta$-氧基化反应，合成 2-(2-氨基芳基)烯氰类化合物 98 (Eq. 21). 作者发现在 $\left.[\mathrm{RhCl}(\mathrm{COD})]_{2}\right] / \mathrm{DPEphos}$ 催化 下, 邻氰胺基芳基乙烯发生 $\mathrm{N}-\mathrm{CN}$ 键断裂并和双键发 生 $\mathrm{H}$ 和 $\mathrm{CN}$ 交换反应生成(2-氨基芳基)氰基乙烯类化合 物.

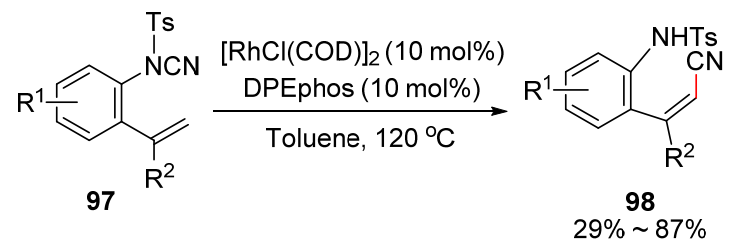

\section{4 缕及其衍生物的合成}

脒为一类既是有多样性生物活性分子, 又是多功能 的有机中间体. 氰胺作为合成榺类化合物的一类重要前
体，已被广泛应用于合成各种脒类化合物。

2007 年 Malacria 和合作者 ${ }^{[52]}$ 报道了 $\mathrm{Bu}_{3} \mathrm{SnH} / \mathrm{AIBN}$ 引发 $N$-酰基氰胺 99 的分子内自由基串联反应高效构建 四环喹唑啉 100, 实现一步构建两个环骨架(Scheme 17). 同时，他们通过对反应条件的调整，成功将该反应应用 于合成天然生物碱 luotonin A. 作者基于底物考察的结 果, 并通过密度泛函理论计算提出了以下反应机理：2碘代苄基氰胺和 $\mathrm{Bu}_{3} \mathrm{SnH} / \mathrm{AIBN}$ 进行反应产生芳基自由 基 $\mathbf{A}, \mathbf{A}$ 发生碳氮参键加成生成㭗亚胺自由基 $\mathbf{B}$. 活性中 间体 $\mathbf{B}$ 进行 6-endo-trig 加成及芳化反应(烯基化合物发 生的是还原反应), 或者 $[1,5]$ 原位取代和重排反应生成 目标产物.

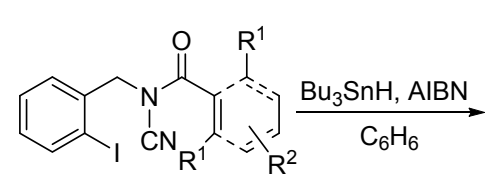

99

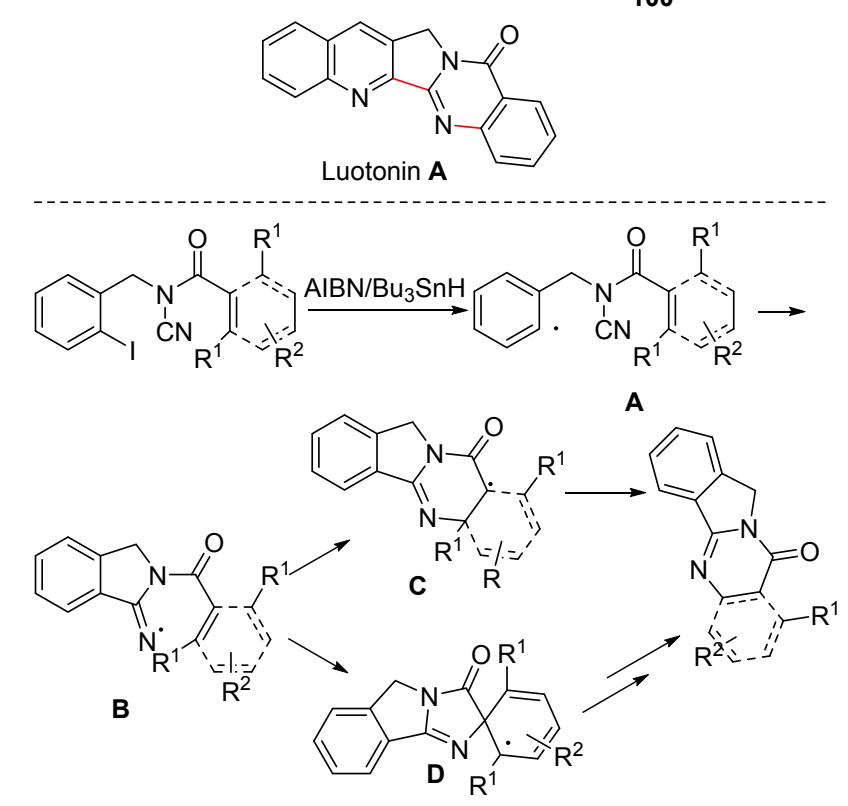

100

图式 $17 N$-酰基氰胺自由基串联反应合成四环稠合喹唑啉 Scheme 17 Radical cascade reaction of $N$-acyl cyanamides for the synthesis of tetracycle-fused quinazolines

几年后，他们 ${ }^{[53]}$ 又发展了 $N$-酰基氰胺(101)的自由 基串联反应合成三环喹唑啉 102 (Scheme 18). 作者对反 应过程进行详细研究, 提出了以下反应机理: $N$-酰基氰 胺和 $\mathrm{Bu}_{3} \mathrm{SnH} / \mathrm{AIBN}$ 发生多步反应产生中间体 $\mathbf{A} . \mathbf{A}$ 发生 6-endo-trig 加成生成 $\mathbf{B} . \mathbf{B}$ 脱去自由基 $\mathrm{R}^{3}$ •生成中间体 $\mathbf{C}$. B 和 $\mathbf{C}$ 之间进行自由基 $\mathrm{R}^{3} \cdot$ 迁移产生 $\mathbf{C}$ 和 D. D 最后发 生自由基氢化生成目标产物.

除了应用于环榺类化合物的合成，通过氰胺反应合 成链状榺类化合物也已有相关报道. 2012 年, Larhed 课 题组 ${ }^{[54]}$ 通过钯催化芳基三氟硼酸钾 103 和㲵胺 104 的 


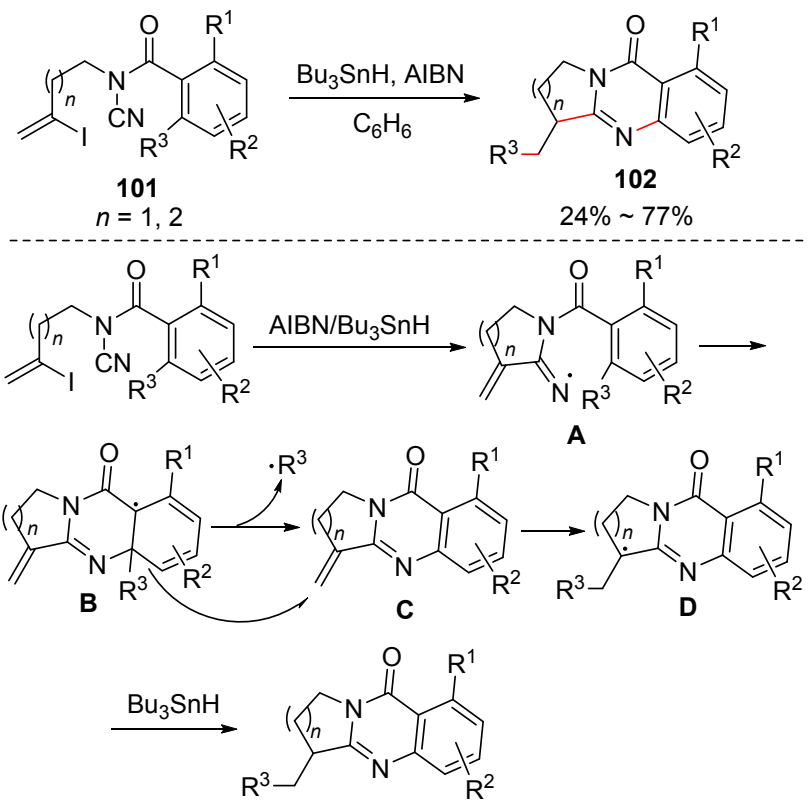

图式 $18 N$-酰基氰胺自由基串联反应合成三环喹唑啉

Scheme 18 Radical cascade reaction of $N$-acyl cyanamides for the synthesis of tricycle-fused quinazolines

加成反应，高效合成芳基榺类化合物 105 (Scheme 19). 他们以三氟醋酸钯为催化剂、6-甲基-2,2'-二吡啶为配体 和三氟乙酸为添加剂, 在微波促进作用下芳基三氟硼酸 钾和㲵胺发生亲核加成反应快速生成芳基脒. 带供电子 基团或者弱拉电子基团的芳基三氟硼酸钾都能参与反 应生中等偏上的产率. 基于实验结果, 作者对反应进行
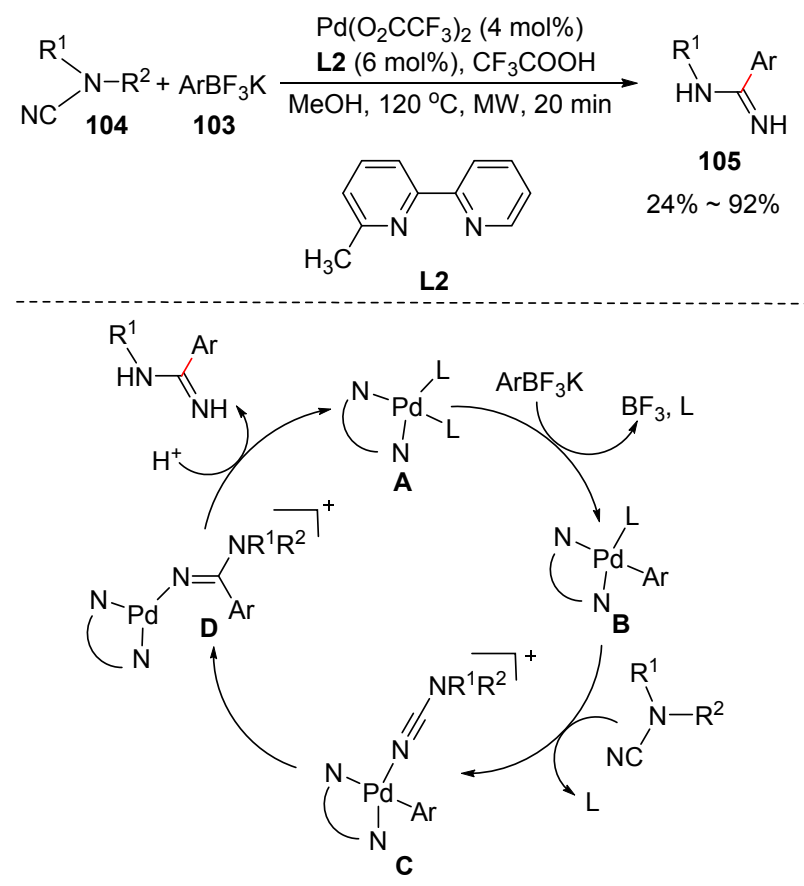

图式 19 钯催化氰胺和芳基三氟嗍酸钾加成反应合成芳基榺 Scheme 19 Palladium-catalyzed addition of cyanamides and potassium arlyltrifluoroborates for the synthesis of arylamidines
推断：二价钯络合物 $\mathbf{A}$ 和芳基三氟硼酸钾发生金属转移 反应产生中间体 B. B 再和氰胺发生配体交换生成 C, C 进行碳氮三键的钯芳基插入反应产生 D. 最后, D 进行 质子化产生目标产物和钯催化剂.

一年后, Larhed 课题组 ${ }^{[55]}$ 又证实了 $\mathrm{Pd}\left(\mathrm{O}_{2} \mathrm{CCF}_{3}\right)_{2} / 6$ 甲基-2,2'-二吡啶催化体系同样可以催化芳基甲酸(106) 和氰胺 107 的脱羧加成反应，生成芳香脒 108 (Eq. 22). 该反应对底物的适用范围有一定的局限性，只有邻位带 有供电子基团的芳香羧酸和脂肪氰胺适应于本反应. 作 者利用 ESI-MS 技术对反应过进行研究, 并通过密度泛 函理论计算，提出了类似芳基三氟嗍酸钾和氰胺反应的 机理(Scheme 19). 芳基甲酸先和二价钯络合物进行配 体交换生成羧基钯，接着发生脱羧反应产生中间体 B. B 再进行配体交换、氭基的钯芳基插入反应和质子化串联 反应即可生成目标产物.

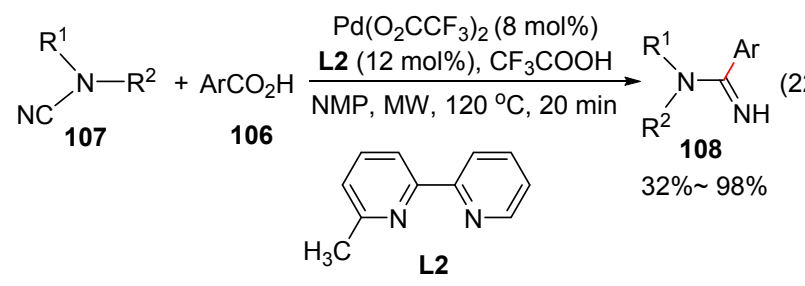

类似地，氰胺还可以应用于合成 $\alpha$-酮酰胺类化合 物. Patel 等 ${ }^{[56]}$ 发展了钯催化氰胺 109 和 $\alpha$-酮酸 110 的串 联反应高效合成 $\alpha$-酮酰胺类化合物 111 (Scheme 20). 在 氧化条件下, $\mathrm{Pd}\left(\mathrm{O}_{2} \mathrm{CCF}_{3}\right)_{2}$ 催化 $\alpha$-酮酸和氰胺发生多步 串联反应生成中等偏上产率的 $\alpha$-酮酰胺类化合物，对卤 素、㲵基等官能团具有良好的兼容性. 通过机理研究, 作者提出以下反应过程: 氰胺和二价钯催化剂配位形成 钯络合物 $\mathbf{A}$, 接着和 $\alpha$-酮酸氧化脱羧产生的酰基自由基 在氧化条件下反应生成四价钯络合物 B. B 进行 1,2-位钯 迁移生成 $\mathbf{C}$, 再进行还原消除反应生成中间体 $\mathbf{D}$ 和钯催 化剂. 最后, D 在酸促进下发生水解反应生成目标产物.

\section{3 结论与展望}

氰胺作为一种具有多样性生物活性的分子和多功 能的精细化工中间体，在医药、农药、材料和染料等领 域都有广泛的应用，激起了合成化学家对其合成方法和 反应活性研究的热情.

氯胺类化合物的合成，经历了由早期直接或者间接 使用剧毒氧化溴原料到近些年来利用低毒性非氰化合 物为原料的绿色发展. 氧胺的合成方法大部分都是基于 传统的两组分反应，近年来通过过渡金属催化多组分反 应合成多样性氰胺类化合物的方法也有报道. 过渡金属 催化多组分反应合成方法高效且具有良好的选择性, 能 


$$
\mathrm{NC}_{109}^{\prime} \underset{110}{\mathrm{RH}+\mathrm{R}^{2} \mathrm{COCO}_{2} \mathrm{H}} \frac{\mathrm{Pd}(\mathrm{TFA})_{2}(10 \mathrm{~mol} \%)}{\left(\mathrm{NH}_{4}\right)_{2} \mathrm{~S}_{2} \mathrm{O}_{8}, \mathrm{DCE}, 80^{\circ} \mathrm{C}} \underset{2 \mathrm{~h}}{\longrightarrow}
$$

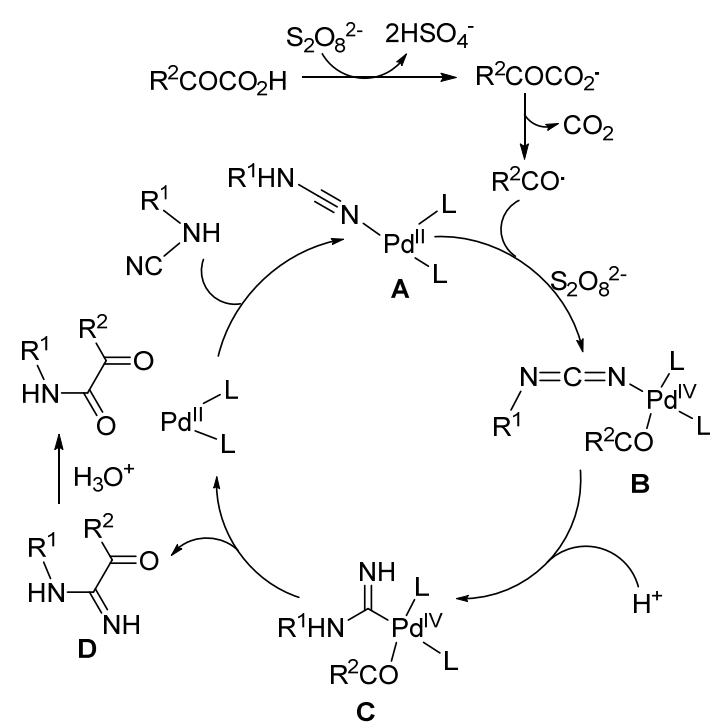

图式 20 钯催化氰胺和 $\alpha$-羰基羧酸脱羧加成反应合成 $\alpha$-酮酰 胺

Scheme 20 Palladium-catalyzed decarboxylative addition of cyanamides and $\alpha$-oxocarboxylic acids for the synthesis of $\alpha$-ketoamides

够实现对结构多样性望胺类的合成，但目前这类合成方 法的研究还处于初级阶段. 随着氧胺在药学和有机合成 领域应用的推广, 对多样性氰胺类化合物有迫切的需 求. 因此, 发展低毒性原料的过渡金属催化多组分反应 实现绿色合成结构多样性氰胺类化合物将是氰胺合成 方法研究的一个重要方向.

随着氰胺合成方法研究的不断推进, 及大的促进了 氯胺反应活性研究的发展. 近 20 年来, 各种氰胺参与的 反应已被发现，高效合成各种含氮化合物，如胍类化合 物、2-氨基吡啶类化合物、榺类化合物和 $\beta$-氧基胺类化 合物等. 这些反应中主要涉及氨基的亲电反应、氧基的 亲核反应或者 N-CN 切断反应等, 氧胺仅作为单性反应 试剂参与反应. 氰胺为含有氨基和氧基双官能团试剂, 具有亲电性和亲核性，可用作双性反应试剂，但其作为 双性反应试剂参与反应的研究工作鲜有报道, 所以以其 作为双性合成子合成多样性含氮化合物的工作有待进 一步挖掘, 也是氰胺反应活性函待研究的重点问题.

\section{Referenes}

[1] Zhu, Y.; Loso, M. R.; Watson, G. B.; Sparks, T. C.; Rogers, R. B.; Huang, J. Z.; Gerwick, B. C.; Babcock, J. M.; Kelley, D.; Hegde, V. B.; Nugent, B. M.; Renga, J. M.; Denholm, I.; Gorman, K.; DeBoer, G. J.; Hasler, J.; Meade, T.; Thomas, J. D. J. Agric. Food Chem. 2011, 59, 2950.
[2] (a) Lainé, D.; Palovich, M.; McCleland, B.; Petitjean, E.; Delhom, I.; Xie, H.; Deng, J.; Lin, G.; Davis, R.; Jolit, A.; Nevins, N.; Zhao, B.; Villa, J.; Schneck, J.; McDevitt, P.; Midgett, R.; Kmett, C.; Umbrecht, S.; Peck, B.; Davis, A. B.; Bettoun, D. ACS Med. Chem. Lett. 2011, 2, 142.

(b) Falgueyrat, J.-P.; Oballa, R. M.; Okamoto, O.; Wesolowski, G.; Aubin, Y.; Rydzewski, R. M.; Prasit, P.; Riendau, D.; Rodan, S. B.; Percival, M. D. J. Med. Chem. 2001, 44, 94.

[3] Feldman, P. L.; Brackeen, M. F.; Cowan, D. J.; Marron, B. E.; Schoenen, F. J.; Stafford, J. A.; Suh, E. M.; Domanico, P. L.; Rose, D.; Leesnitzer, M. A.; Brawley, E. S.; Strickland, A. B.; Vergese, M. W.; Connolly, K. M.; Bateman-Fite, R.; Noel, S. L.; Sekut, L.; Stimpson, S. A. J. Med. Chem. 1995, 38, 1505.

[4] (a) Larraufie, M. H.; Maestri, G.; Malacria, M.; Ollivier, C.; Fensterbank, L.; Lacote, E. Synthesis 2012, 44, 1279. (b) Nekrasov, D. D. Russ. J. Org. Chem. 2004, 40, 1387.

[5] Crutchley, R. J. Coord. Chem. Rev. 2001, 219, 125.

[6] Boatright, L. G.; Mackay, J. S. US 2721786, 1955 [Chem. Abstr. 1956, 50, 21846].

[7] (a) Nekrasov, D. D. Russ. J. Org. Chem. 2004, 40, 1387. (b) Larraufie, M. H.; Maestri, G.; Malacria, M.; Ollivier, C.; Fensterbank, L.; Lacote, E. Synthesis 2012, 44, 1279.

[8] Braun, von J. Ber. Dtsch. Chem. Ges. 1907, 40, 3914.

[9] Morgan, T.; Ray, N. C.; Parry, D. M. Org. Lett. 2002, 4, 597.

[10] Nath, J.; Patel, B. K.; Jamir, L.; Sinha, U. B.; Satyanarayan, K. V. V. V. Green Chem. 2009, 11, 1503

[11] Ramana, T.; Saha, P.; Das, M.; Punniyamurthy, T. Org. Lett. 2010 , 12,84

[12] Sahoo, S. K.; Jamir, L.; Guin, S.; Patel, B. K. Adv. Synth. Catal. 2010, 352, 2538.

[13] Zhu, C.; Xia, J.-B.; Chen, C. Org. Lett. 2014, 16, 247.

[14] Lin, C.-C.; Hsieh, T.-H.; Liao, P.-Y.; Liao, Z.-Y.; Chang, C.-W.; Shih, Y.-C.; Yeh, W.-H.; Chien, T.-C. Org. Lett. 2014, 16, 892.

[15] Ayres, J. N.; Ling, K. B.; Morrill, L. C. Org. Lett. 2016, 18, 5528.

[16] Kamijo, S.; Jin, T.; Yamamoto, Y. J. Am. Chem. Soc. 2001, 123, 9453.

[17] Kamijo, S.; Yamamoto, Y. J. Am. Chem. Soc. 2002, 124, 11940.

[18] Kamijo, S.; Jin, T. Yamamoto, Y. Angew. Chem., Int. Ed. 2002, 41, 1780.

[19] Stolley, R. M.; Guo, W.; Louie, J. Org. Lett. 2012, 14, 322.

[20] Li, P.; Cheng, G.; Zhang, H.; Xu, X.; Gao, J.; Cui, X. J. Org. Chem. 2014, 79, 8156.

[21] Li, J.; Zheng, X.; Li, W.; Zhou, W.; Zhu W.; Zhang, Y. New J. Chem. 2016, 40, 77.

[22] (a) Reddy, N. L.; Fan, W.; Magar, S. S.; Perlman, M. E.; Yost, E.; Zhang, L.; Berlove, D.; Fischer, J. B.; Burke-Howie, K.; Wolcott, T.; Durant, G. J. J. Med. Chem. 1998, 41, 3298.

(b) Snider, B. B.; O'Hare, S. M. Tetrahedron Lett. 2001, 42, 2455.

(c) Basterfield, S.; Rodman, F. B. S.; Tomecko J. W. Can. J. Res. 2011, 17, 390

[23] Giles, R. L.; Sullivan, J. D.; Steiner, A. M.; Looper, R. E. Angew. Chem., Int. Ed. 2009, 48, 3116.

[24] Giles, R. L.; Nkansah, R. A.; Looper, R. E. J. Org. Chem. 2010, 75 261.

[25] Lou, Z.; Wu, X.; Yang, H.; Zhu, C.; Fu, H. Adv. Synth. Catal. 2015, 357,3961 .

[26] Rassadin, V. A.; Zimin, D. P.; Raskil'dina, G. Z.; Ivanov, A. Y.; Boyarskiy, V. P.; Zlotskiib S. S.; Kukushkin, V. Y. Green Chem. 2016, 18,6630 .

[27] Larraufie, M.-H.; Ollivier, C.; Fensterbank, L.; Malacria, M.; Lacte, E. Angew. Chem., Int. Ed. 2010, 49, 2178

[28] Maestri, G.; Larraufie, M.-H.; Ollivier, C.; Malacria, M.; Fensterbank, L.; Lacôte, E. Org. Lett. 2012, 14, 5538.

[29] Li, J.; Neuville, L. Org. Lett. 2013, 15, 6124.

[30] Tran, L. Q.; Li, J.; Neuville, L. J. Org. Chem. 2015, 80, 6102.

[31] Li, J.; Wang, H.; Hou, Y.; Yu, W.; Xu, S.; Zhang, Y. Eur. J. Org. Chem. 2016, 2388.

[32] Vollhardt, K. P. C.; Naiman, A. US 4328343, 1982 [Chem. Abstr. 
1978, 90, 186806].

[33] Boñaga, L. V. R.; Zhang H.-C.; Maryanoff B. E. Chem. Commun. 2004, 2394.

[34] (a) Stolley, R. M.; Maczka, M. T.; Louie J. Eur. J. Org. Chem. 2011, 3815.

(b) Kumar, P.; Prescher, S.; Louie, J. Angew. Chem., Int. Ed. 2011, 50, 10694.

[35] Wang, C.; Wang, D.; Xu, F.; Pan, B.; Wan, B. J. Org. Chem. 2013, $78,3065$.

[36] Lane, T. K.; D’Souza, B. R.; Louie, J. J. Org. Chem. 2012, 77, 7555.

[37] Lane, T. K.; Nguyen, M. H.; D’Souza, B. R.; Spahn, N. A.; Louie, J. Chem. Commun. 2013, 49, 7735.

[38] Hashimoto, T.; Ishii, S.; Yano, R.; Miura, H.; Sakata, K.; Takeuchi, R. Adv. Synth. Catal. 2015, 357, 3901.

[39] Onodera, G.; Shimizu, Y.; Kimura, J.; Kobayashi, J.; Ebihara, Y.; Kondo, K.; Sakata, K.; Takeuchi, R. J. Am. Chem. Soc. 2012, 134, 10515.

[40] Ye, F.; Haddad, M.; Ratovelomanana-Vidal, V.; Michelet, V. Org. Lett. 2017, 19, 1104

[41] Rassadin, V. A.; Boyarskiy, V. P.; Kukushkin Y. V. Org. Lett. 2015, 17,3502 .

[42] (a) Hughes, T. V.; Hammond, S. D.; Cava, M. P. J. Org. Chem. 1998, 63, 401

(b) Hughes, T. V.; Cava, M. P. J. Org. Chem. 1999, 64, 313.

[43] Wu, Y.-Q.; Limburg, D. C.; Wilkinson, D. E.; Hamilton, G. S. Org. Lett. 2000, $2,795$.

[44] Anbarasan, P.; Neumann, H.; Beller, M. Chem.-Eur. J. 2010, 16, 4725.

[45] (a) Anbarasan, P.; Neumann, H.; Beller, M. Angew. Chem. Int. Ed. 2011, 50, 519 .

(b) Anbarasan, P.; Neumann, H.; Beller, M. Chem.-Eur. J. 2011, 17, 4217.

(c) Cai, Y.; Qian, X.; Rérat, A.; Auffrant, A.; Gosmini C. Adv. Synth. Catal. 2015, 357, 3419

(d) Yang, Y.; Zhang, Y.; Wang, J. Org. Lett. 2011, 13, 5608. (e) Gong, T.-J.; Xiao, B.; Cheng, W.-M.; Su, W.; Xu, J.; Liu, Z.-J.; Liu, L.; Fu, Y. J. Am. Chem. Soc. 2013, 135, 10630.

(f) Chaitanya, M.; Yadagiri, D.; Anbarasan, P. Org. Lett. 2013, 15, 4960.

(g) Gu, L.-J.; Jin, C.; Wang, R.; Ding, H.-Y. ChemCatChem 2014, 6,1225 .

(h) Mishra, N. K.; Jeong, T.; Sharma, S.; Shin, Y.; Han, S.; Park, J.; Oh, J. S.; Kwak, J. H.; Jung, Y. H.; Kima, I. S. Adv. Synth. Catal. 2015, 357, 1293 .

(i) Chaitanya, M.; Anbarasan, P. J. Org. Chem. 2015, 80, 3695.

(j) Zhu, X.; Shen, X.J.; Tian, Z.-Y.; Lu, S.; Tian, L.-L.; Liu, W.-B.; Song, B.; Hao, X.-Q. J. Org. Chem. 2017, 82, 6022.

[46] Fukumoto, K.; Oya, T.; Itazaki, M.; Nakazawa, H. J. Am. Chem. Soc. 2009, 131, 38.

[47] Liao, Z.-Y.; Liao, P.-Y.; Chien, T.-C. Chem. Commun. 2016, 52, 14404.

[48] Rao, B.; Zeng, X. Org. Lett. 2014, 16, 314

[49] Pan, Z.; Pound, S. M.; Rondla, N. R.; Douglas, C. J. Angew. Chem., Int. Ed. 2014, 53, 5170.

[50] Miyazaki, Y.; Ohta, N.; Semba, K.; Nakao, Y. J. Am. Chem. Soc. 2014, 136, 3732

[51] Wang, R.; Falck, J. R. Chem. Commun. 2013, 49, 6516.

[52] (a) Servais, A.; Azzouz, M.; Lopes, D.; Courillon, C.; Malacria, M. Angew. Chem., Int. Ed. 2007, 46, 576.

(b) Beaume, A.; Christine Courillon, C.; Derat, E.; Malacria, M. Chem.-Eur. J. 2008, 14, 1238.

[53] Larrafie, M.-H.; Courillon, C.; Ollivier, C.; Lacôte, E.; Malacria, M.; Fensterbank, L. J. Am. Chem. Soc. 2010, 132, 4381.

[54] Sävmarker, J.; Rydfjord, J.; Gising, J.; Odell, L. R.; Larhed, M. Org. Lett. 2012, 14, 2394.

[55] Rydfjord, J.; Svensson, F.; Trejos, A.; Söjberg, P. J. R.; Sköld, C.; Sävmarker, J.; Odell, L. R.; Larhed, M. Chem.-Eur. J. 2013, 19 , 13803.

[56] Guin, S.; Rout, S. K.; Gogoi, A.; Ali, W.; Patel, B. K. Adv. Synth. Catal. 2014, 46, 2559. 